\title{
Mobility Variation and Kinematics Analysis of a Family of Metamorphic Parallel Mechanisms with Reconfigurable Hybrid Limbs
}

\author{
Wei Ye ${ }^{\text {a }}$, Yuefa Fang ${ }^{\text {a, }}{ }^{\text {, }}$ Ketao Zhang ${ }^{\text {a, b }}$, Sheng Guo ${ }^{\text {a }}$ \\ ${ }^{a}$ Department of Mechanical Engineering, Beijing Jiaotong University, Beijing 100044, P.R. China \\ ${ }^{\mathrm{b}}$ Centre for Robotics Research, Faculty of Natural and Mathematical Sciences, King's College London, University of London, \\ London WC2R 2LS, UK
}

\section{ABSTRACT}

This paper presents a planar metamorphic mechanism (PMM) which has three distinct working phases and a family of metamorphic parallel mechanisms with a PMM as closed-loop subchain in each limb. The study starts from analysis of mobility variation and motion characteristics in each phase of the PMM based on screw theory. Three types of reconfigurable hybrid limbs are then constructed by integrating the PMM and serial chains capable of supplying one constraint force and one constraint couple. This leads to the design of a new family of metamorphic parallel mechanisms in which the platform is connected to the base by three reconfigurable hybrid limbs. The configuration changes of the reconfigurable limb associate with the three distinct phases of the PMM are analyzed and the constraints exerted by the reconfigurable limb in various configurations are identified. The analyses reveal that the metamorphic parallel mechanisms have ability to alter the performance of platform from full 6-DOF configuration to 5-, 4- and 3-DOF configurations resorting to the internal configuration variation of the three reconfigurable limbs. Finally, Actuation models with selected joints for mounting drives for the metamorphic parallel mechanisms are addressed and application cases including the potential for hybrid additive and subtractive manufacturing machine of the proposed mechanisms are discussed in details.

KEYWORDS: planar metamorphic mechanism, reconfigurable hybrid limb, metamorphic parallel mechanism, screw theory, configuration variation, actuation scheme

\section{Introduction}

With the rapid development of science and technology, robots and mechanisms with changeable structures and mobility are expected in production to accommodate different operation environments and meet various task requirements. Several kinds of reconfigurable mechanisms such as the kinematotropic linkages $[1,2]$ and metamorphic mechanisms $[3,4]$ have been developed in the past decades.

${ }^{*}$ Corresponding author (email: yffang@bjtu.edu.cn) 
Metamorphic mechanisms are a kind of mechanical assemblies with changeable mobility resorting to their structure variations. Since the proposal in 1998 [5], the metamorphic mechanisms have drawn more and more attentions in the field of mechanisms and robotics. Parise et al. [6] developed ortho-planar metamorphic mechanisms which can change their topological structures in two orthogonal planes. Chen et al. [7] proposed a metamorphic underwater vehicle that can change the shape of its body continuously in accordance with the microorganism. Liu and Yang [8] investigated the essence and characteristics of metamorphic mechanisms and presented three metamorphic ways for the topological structure changes of metamorphic mechanisms. Dai and Jones [9] introduced matrix representation for changing the topological structures of metamorphic mechanisms. Wang and Dai [10] presented an metamorphic equation for representing the configuration change of metamorphic mechanisms. Dai et al. [11-12] presented a novel multi-fingered robotic hand in which a five-bar spherical linkage is used as metamorphic palm. Li et al. [13] developed a systematic methodology for the structure synthesis of metamorphic mechanisms based on augmented Assur groups. Valsamos et al. [14] proposed an approach to determine the best anatomy of a metamorphic serial linkage for a given task.

Though the metamorphic mechanisms have been extensively studied, relative few metamorphic parallel mechanisms with ability either to alter motion mode or to perform mobility change were presented [15]. Zhang et al. [16,17] proposed a vA joint which has three mobility phases and integrated the vA joint in the construction of several metamorphic parallel mechanisms. Gan et al. [18-21] presented a new joint coined as rT joint and put forward a general procedure for mobility-change-aimed metamorphic parallel mechanism construction. Carbonari et.al [22-25] designed a lockable universal joint and developed a class of metamorphic parallel mechanisms which can be used in many applications such as in quality control or haptic interface. Apart from the metamorphic parallel mechanisms, the kinematotropic parallel mechanisms, which also constitute an important class of reconfigurable parallel mechanisms, have attracted certain attentions in the research community. Several kinematotropic parallel mechanisms which employ the constraint singularities either among the serial limbs [26-29], or inside the hybrid limbs due to the existence of kinematotropic closed-loop chains [30], have been proposed. It should be noticed that in order to keep favourable dynamic performance, the actuation scheme of reconfigurable mechanisms with parallel structures should be taken into consideration in the design stage.

In this paper, a planar metamorphic mechanism (PMM) with three distinct phases is presented and then integrated in the construction of three types of reconfigurable limbs leading to a new family of metamorphic parallel mechanisms. The wide range mobility variations of the proposed metamorphic parallel mechanisms are addressed and the superior actuation schemes for the mechanisms are demonstrated. This paper is arranged as follows: section 2 puts forward the planar metamorphic mechanism and its phase change. A new family of metamorphic parallel mechanisms and their basic characteristics are presented in section 3. In section 4 , topological configurations and constraints of the $\mathrm{R}_{1} \mathrm{PR}_{1} \mathrm{R}_{2}-\mathrm{PMM}$ reconfigurable limb are analyzed. Mobility variations 
of the $3 R_{1} P R_{1} R_{2}-P M M$ parallel mechanism are demonstrated in section 5. Section 6 analyzes the actuation scheme and discusses the advantages and potential applications of the proposed mechanisms. Finally, conclusions are drawn.

\section{A Planar Metamorphic Mechanism (PMM) and the Motion Analysis}

Recently, a structure synthesis methodology of planar single driven metamorphic mechanisms based on the augmented Assur groups was developed and a number of planar metamorphic mechanisms were constructed by Li and Dai [13]. The two distinct phases of a typical planar metamorphic mechanism are shown in Fig. 1. This mechanism is a planar five bar linkage in which the slide link 3 is connected to sleeve 4 with spring but has only one actuator mounted to joint $A$. The mechanism in Fig. 1(a) is a working phase with one degree of freedom (DOF) since the relative motion between slide link 3 and sleeve 4 is constrained by the spring. The mechanism works as a planar four bar linkage with four revolute joints $A, B, C$ and $E$ with parallel axes. When the mechanism moves to the phase in Fig. 1(b), link 1 and link 2 are annexed and the joint $B$ is physically constrained. The relative motion between slide link 3 and sleeve 4 becomes effective when the actuated joint $A$ rotating anticlockwise. Under such a condition, the mechanism evolves to a crank-slider mechanism with effective joints $A, C, D$ and $E$.

\subsection{Design of a PMM with Three Working Phases}

Inspired by the mechanism in Fig. 1, a modified planar metamorphic mechanism denoted in short by PMM is proposed and the three distinct phases of this PMM are illustrated in Fig. 2. The spring between slide link 3 and sleeve 4 in Fig. 1 is removed and the mechanism has two degrees of freedom in its source phase in Fig. 2(a) and two motors are mounted to joints $A$ and $D$ respectively to fully actuate the mechanism. When the mechanism moves to the phase in Fig. 2(b) where the joint $D$ is locked by shrinking back the prismatic joint to its shortest stroke, the mechanism changes to one DOF with one effective actuator at joint $A$ and works as a equivalent planar four bar linkage with four revolute joints $A, B, C$ and $E$. Taking into account of the unique characteristics of planar four-bar parallelogram which has been employed in the construction of several distinguished parallel mechanisms [31,32], the parameters of the PMM in Fig. 2(b) are specifically defined to satisfy $|A B|=|C E|$ and $|B C|=|A E|$ which results in the evolved planar four bar parallelogram denoted with $\mathrm{P}$ phase. When the mechanism moves to another configuration in Fig. 2(c) where the axes of three revolute joints $A, B$ and $E$ are coplanar and the link 1 are locally restricted with respect to link 5, the five-bar linkage changes to a crank-slider mechanism and is denoted with $\mathrm{R}$ phase. In this configuration, revolute joint $A$ is locked and only the actuator mounted to prismatic joint $D$ is effective.

Hence, the PMM has three phases including the source phase, $\mathrm{P}$ phase and $\mathrm{R}$ phase. The source phase is a transferring phase and both the $\mathrm{P}$ phase and $\mathrm{R}$ phase can return to the source phase with two active actuators at joints $A$ and $D$. 


\subsection{Equivalent Motion Screws of the PMM in Different Phases}

Since the PMM has three distinct working phases, the exact motion characteristics of this planar closed-loop chain corresponding to each phase are analyzed in this section based on screw theory [33-35].

Attach a local coordinate system $\left\{x_{i} y_{i} z_{i}\right\}$ to the PMM with its original point located at the center of joint $A$ as in Fig. 3 . The $x_{i^{-}}$ axis is aligned with axis of joint $A$ and $y_{i}$-axis is perpendicular to $x_{i}$ axis and lies in the plane formed by axes of revolute joints $A$ and $E$, the $z_{i}$ axis is defined according to the right-hand rule. The output link 2 is connected to the base link 5 with branches 1 and 2, the motion screw systems $\left\{\mathbf{S}_{b 1}\right\}$ and $\left\{\mathbf{S}_{b 2}\right\}$ of these two branches are given by

$$
\begin{aligned}
& \left\{\mathbf{S}_{b 1}\right\}=\left\{\begin{array}{l}
\mathbf{S}_{b 11}=\left[\begin{array}{llllll}
1 & 0 & 0 & 0 & 0 & 0
\end{array}\right]^{\mathrm{T}} \\
\mathbf{S}_{b 12}=\left[\begin{array}{llllll}
1 & 0 & 0 & 0 & z_{\mathrm{b}} & -y_{\mathrm{b}}
\end{array}\right]^{\mathrm{T}}
\end{array}\right. \\
& \left\{\mathbf{S}_{b 2}\right\}=\left\{\begin{array}{l}
\mathbf{S}_{b 21}=\left[\begin{array}{lllll}
1 & 0 & 0 & 0 & z_{\mathrm{e}}-y_{\mathrm{e}}
\end{array}\right]^{\mathrm{T}} \\
\mathbf{S}_{b 22}=\left[\begin{array}{llllll}
0 & 0 & 0 & 0 & y_{\mathrm{c}}-y_{\mathrm{e}} z_{\mathrm{c}}-z_{\mathrm{e}}
\end{array}\right]^{\mathrm{T}} \\
\mathbf{S}_{b 23}=\left[\begin{array}{llllll}
1 & 0 & 0 & 0 & z_{\mathrm{c}}-y_{\mathrm{c}}
\end{array}\right]^{\mathrm{T}}
\end{array}\right.
\end{aligned}
$$

where $\left[\begin{array}{lll}0 & y_{\mathrm{b}} & z_{\mathrm{b}}\end{array}\right]^{\mathrm{T}},\left[\begin{array}{lll}0 & y_{\mathrm{c}} & z_{\mathrm{c}}\end{array}\right]^{\mathrm{T}}$ and $\left[\begin{array}{lll}0 & y_{\mathrm{e}} & z_{\mathrm{e}}\end{array}\right]^{\mathrm{T}}$ are the position vectors of the center point of joint $B, C$ and $E$ in the local coordinate system $\left\{x_{i} y_{i} z_{i}\right\}$.

Based on the motion screw systems, the basis of the constraint screw system $\left\{\mathbf{S}_{b}^{r}\right\}$ exerted on the output link 2 by branches 1 and 2 can be yielded as

$$
\left\{\mathbf{S}_{b}^{r}\right\}=\left\{\begin{array}{l}
\mathbf{S}_{b 1}^{r}=\left[\begin{array}{llllll}
1 & 0 & 0 & 0 & 0 & 0
\end{array}\right]^{\mathrm{T}} \\
\mathbf{S}_{b 2}^{r}=\left[\begin{array}{llllll}
0 & 0 & 0 & 0 & 1 & 0
\end{array}\right]^{\mathrm{T}} \\
\mathbf{S}_{b 3}^{r}=\left[\begin{array}{llllll}
0 & 0 & 0 & 0 & 0 & 1
\end{array}\right]^{\mathrm{T}} \\
\mathbf{S}_{b 4}^{r}=\left[\begin{array}{llllll}
0 & y_{\mathrm{b}} & z_{\mathrm{b}} & 0 & 0 & 0
\end{array}\right]^{\mathrm{T}}
\end{array}\right.
$$

The possible motion screws of the output link 2 are reciprocal to the constraint screw system and can be calculated as

$$
\left\{\mathbf{S}_{\mathrm{PMM}}\right\}=\left\{\begin{array}{l}
\mathbf{S}_{i 1}=\left[\begin{array}{llllll}
0 & 0 & 0 & 0 & -z_{\mathrm{b}} & y_{\mathrm{b}}
\end{array}\right]^{\mathrm{T}} \\
\mathbf{S}_{i 2}=\left[\begin{array}{llllll}
1 & 0 & 0 & 0 & 0 & 0
\end{array}\right]^{\mathrm{T}}
\end{array}\right.
$$

The above two motion screws present one rotational motion around $x_{i}$-axis and one translational motion in $y_{i} o_{i} z_{i}$ plane.

When the PMM changes its configuration to the P phase in Fig. 4, joint $D$ is locked and the motion screw system $\left\{\mathbf{S}_{b 2}\right\}$ of branch 2 becomes

$$
\left\{\mathbf{S}_{b 2}\right\}=\left\{\begin{array}{l}
\mathbf{S}_{b 21}=\left[\begin{array}{lllll}
1 & 0 & 0 & 0 & z_{\mathrm{e}}-y_{\mathrm{e}}
\end{array}\right]^{\mathrm{T}} \\
\mathbf{S}_{b 23}=\left[\begin{array}{llllll}
1 & 0 & 0 & 0 & z_{\mathrm{e}}+z_{\mathrm{b}}-y_{\mathrm{e}}-y_{\mathrm{b}}
\end{array}\right]^{\mathrm{T}}
\end{array}\right.
$$

The motion screw system $\left\{\mathbf{S}_{b 1}\right\}$ of branch 1 remains and one of the bases of the constraint screw system $\left\{\mathbf{S}_{b}^{r}\right\}$ exerted on the output link 2 by branches 1 and 2 is given by 


$$
\left\{\mathbf{S}_{b}^{r}\right\}=\left\{\begin{array}{l}
\mathbf{S}_{b 1}^{r}=\left[\begin{array}{llllll}
1 & 0 & 0 & 0 & 0 & 0
\end{array}\right]^{\mathrm{T}} \\
\mathbf{S}_{b 2}^{r}=\left[\begin{array}{llllll}
0 & 0 & 0 & 0 & 1 & 0
\end{array}\right]^{\mathrm{T}} \\
\mathbf{S}_{b 3}^{r}=\left[\begin{array}{llllll}
0 & 0 & 0 & 0 & 0 & 1
\end{array}\right]^{\mathrm{T}} \\
\mathbf{S}_{b 4}^{r}=\left[\begin{array}{llllll}
0 & y_{\mathrm{b}} & z_{\mathrm{b}} & 0 & 0 & 0
\end{array}\right]^{\mathrm{T}} \\
\mathbf{S}_{b 5}^{r}=\left[\begin{array}{llllll}
0 & y_{\mathrm{b}} & z_{\mathrm{b}} & y_{\mathrm{e}} z_{\mathrm{b}}-y_{\mathrm{b}} z_{\mathrm{e}} & 0 & 0
\end{array}\right]^{\mathrm{T}}
\end{array}\right.
$$

The possible motion screw system of the output link 2 in this configuration is then derived by calculating the reciprocal screws of Eq. (6), that is

$$
\left\{\mathbf{S}_{\mathrm{PMM}}\right\}=\mathbf{S}_{i p}=\left[\begin{array}{llllll}
0 & 0 & 0 & 0 & -z_{\mathrm{b}} & y_{\mathrm{b}}
\end{array}\right]^{\mathrm{T}}
$$

This motion screw presents a pure translational motion in $y_{i} o_{i} z_{i}$ plane.

When the PMM changes to the $\mathrm{R}$ phase in Fig. 5, the motion of joint $A$ is physically restricted and the output motion of link 2 is a rotational motion around joint $B$. The equivalent motion screw of the output link is given by

$$
\left\{\mathbf{S}_{\mathrm{PMM}}\right\}=\mathbf{S}_{i r}=\left[\begin{array}{llllll}
1 & 0 & 0 & 0 & 0 & -d_{1}
\end{array}\right]^{\mathrm{T}}
$$

where $d_{1}$ is the length of link 1 .

\section{Structural Composition of Reconfigurable Hybrid Limbs and Metamorphic Parallel Mechanisms}

By integrating the PMM in the structure design of hybrid kinematic chains, a class of metamorphic limbs with reconfigurablity induced by phase changes of the PMM can be constructed. Using three identical reconfigurable hybrid limbs as chain legs that connect the platform to the base, a new family of metamorphic parallel mechanisms can be constructed and are enumerated in Table 1.

In Table 1, $\mathrm{R}$ denotes revolute joint, $\mathrm{P}$ denotes prismatic joint, $\mathrm{U}$ denotes universal joint (equivalent to two revolute joints with intersecting axis) and $\mathrm{C}$ denotes cylinder joint (equivalent to a pair of coaxial revolute and prismatic joints). The types of limbs are represented by the number of $\mathrm{R}$ and $\mathrm{P}$ joints in the limb in sequence and the metamorphic parallel mechanisms of this family have three reconfigurable limbs symmetrically arranged. For example, the sketch of a $3 R_{1} P_{1} R_{2}-P M M$ metamorphic parallel mechanism is illustrated in Fig. 6.

With reference to the types of the limb and structure of the parallel mechanisms in Table 1 and a representative mechanism in Fig. 6 and Fig.7, the characteristics of the metamorphic parallel mechanisms in this family are summarized as follows:

1. The platform is connected to the base through three identical reconfigurable hybrid limbs.

2. Each reconfigurable hybrid limb consists of a PMM as closed-loop subchain and a serial subchain.

3. The serial subchain is selected from a class of kinematic chains which have four DOFs and generate a constraint force and a constraint couple. In the serial subchain, $R_{1}$ represents a revolute joint with axis parallel to the constraint force and $R_{2}$ 
represents a revolute joint with axis intersecting to the constraint force, $\mathrm{P}$ represents a prismatic joint with axis perpendicular to the constraint force. The revolute joints denoted by $R_{1}$ and $R_{2}$ form two sets of parallel axes whose common normal defines the direction of the constraint couple.

4. When the PMM subchain in each limb changes its configuration to $\mathrm{R}$ phase, the axes of joints $\mathrm{R}_{i 2}$ and $B_{i}(i=1,2,3)$ intersect at a common point $O$ in Fig. 7.

\section{Topological Phases and Constraints of the $\mathbf{R}_{\mathbf{1}} \mathbf{P R}_{\mathbf{1}} \mathbf{R}_{\mathbf{2}}-\mathrm{PMM}$ Reconfigurable Limb}

The metamorphic parallel mechanisms enumerated in Table 1 have the ability to change their mobility resorting to the phase change of the PMMs in the reconfigurable hybrid limbs. This section is going to analyze the mobility variation of the $3 R_{1} P R_{1} R_{2}-$ PMM metamorphic parallel mechanism and demonstrate the reconfiguration. Each reconfigurable hybrid limb of the metamorphic parallel mechanism consists of a $P M M$ subchain and a $\mathrm{R}_{1} \mathrm{PR}_{1} \mathrm{R}_{2}$ serial subchain as illustrated in Fig. 6. The PMM subchain leads to three distinct configurations of each reconfigurable hybrid limb. The constraints delivered by the $\mathrm{R}_{1} \mathrm{PR}_{1} \mathrm{R}_{2}-\mathrm{PMM}_{\mathrm{M}}$ reconfigurable hybrid limb in different configurations will be analyzed based on screw theory and the concept of equivalent motion screws [36].

\subsection{Constraint of Limb Configuration $\mathbf{R}_{1} \mathrm{PR}_{1} \mathbf{R}_{2}-\mathrm{PMM}_{\mathrm{S}}$}

The source limb configuration $\mathrm{R}_{1} \mathrm{PR}_{1} \mathrm{R}_{2}-\mathrm{PMM}_{\mathrm{s}}$ with the PMM in its source phase is denoted by the subscript ' $\mathrm{s}$ ' and illustrated in Fig. 8.

In this configuration, the equivalent output motions of the PMM in its source phase are represented by $\mathbf{S}_{i 1}$ and $\mathbf{S}_{i 2}$ in Eq. (4). Integrating the screws for equivalent motion of the closed-loop subchain, the motion screw system of the reconfigurable hybrid limb expressed in the local coordinate system $\left\{x_{i} y_{i} z_{i}\right\}$ is given by

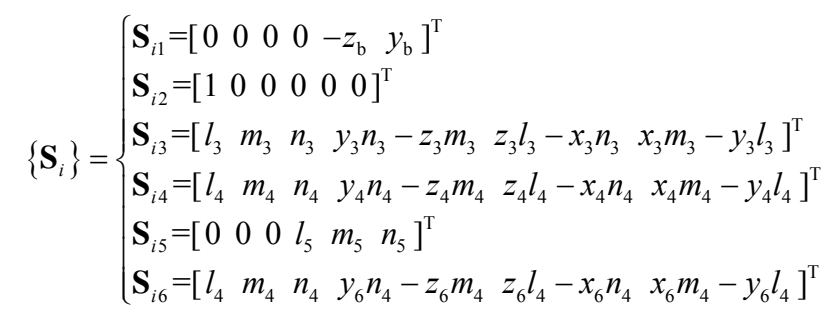

where $\left[\begin{array}{lll}l_{3} & m_{3} & n_{3}\end{array}\right]^{\mathrm{T}}$ and $\left[\begin{array}{llll}l_{5} & m_{5} & n_{5}\end{array}\right]^{\mathrm{T}}$ are the unit vectors pointing in the direction of screws $\mathbf{S}_{i 3}$ and $\mathbf{S}_{i 5} \cdot\left[\begin{array}{lllll}l_{4} & m_{4} & n_{4}\end{array}\right]^{\mathrm{T}}$ is a unit vector pointing in the direction of screws $\mathbf{S}_{i 4}$ and $\mathbf{S}_{i 6}$ and vector $\left[\begin{array}{lll}l_{4} & m_{4} & n_{4}\end{array}\right]^{\mathrm{T}}$ is perpendicular to vector $\left[\begin{array}{lll}l_{5} & m_{5} & n_{5}\end{array}\right]^{\mathrm{T}}$. $\left[\begin{array}{lll}x_{3} & y_{3} & z_{3}\end{array}\right]^{\mathrm{T}},\left[\begin{array}{lll}x_{4} & y_{4} & z_{4}\end{array}\right]^{\mathrm{T}}$ and $\left[\begin{array}{lll}x_{6} & y_{6} & z_{6}\end{array}\right]^{\mathrm{T}}$ are the position vectors of three points on the axes of screws $\mathbf{S}_{i 3}, \mathbf{S}_{i 4}$ and $\mathbf{S}_{i 6}$ respectively. 
The motion screws in Eq. (9) are independent and there is no reciprocal screw corresponding to the motion screw system. This results in constraint free of the platform by the reconfigurable hybrid limb in Fig. 8.

\subsection{Constraint of Limb Configuration $R_{1} P_{1} R_{2}-P M M_{P}$}

The limb configuration $\mathrm{R}_{1} \mathrm{PR}_{1} \mathrm{R}_{2}-\mathrm{PMM}_{\mathrm{P}}$ is illustrated in Fig. 9 where the PMM changes its configuration to the $\mathrm{P}$ phase. The equivalent motion screw of the PMM is given by $\mathbf{S}_{i p}$ in Eq. (7) and the motion screw system of the reconfigurable hybrid limb expressed in the local coordinate system $\left\{x_{i} y_{i} z_{i}\right\}$ becomes

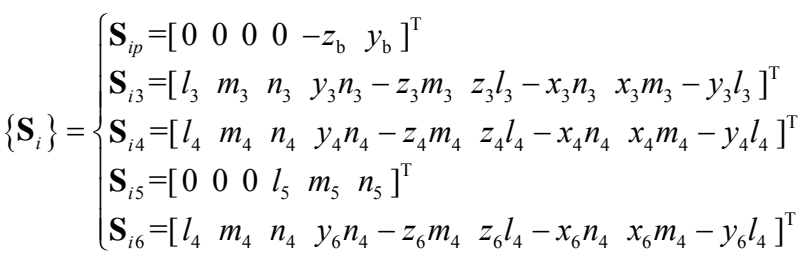

The motion screws form a 5-system and a reciprocal constraint screw can be yielded as

$$
\left\{\mathbf{S}_{i}^{r}\right\}=\mathbf{S}_{i 1}^{r}=\left[\begin{array}{lllllll}
0 & 0 & 0 & m_{3} n_{4}-m_{4} n_{3} & n_{3} l_{4}-l_{3} n_{4} & l_{3} m_{4}-m_{3} l_{4}
\end{array}\right]^{\mathrm{T}}
$$

The above constraint screw presents a constraint couple which is perpendicular to axes of screws $\mathbf{S}_{i 3}, \mathbf{S}_{i 4}$ and $\mathbf{S}_{i 6}$.

\subsection{Constraint of Limb Configuration $R_{1} P_{2} R_{2}-P M M_{R}$}

When the PMM changes its configuration to the R phase in Fig. 5, the reconfigurable hybrid limb changes to $R_{1} P R_{1} R_{2}-P_{M M}$ configuration and is illustrated in Fig. 10. Since the axis of the equivalent motion screw $\mathbf{S}_{i r}$ in Eq. (8) and the axis of screw $\mathbf{S}_{i 3}$ have common point $O$, the motion screw system of the limb configuration $\mathrm{R}_{1} \mathrm{PR}_{1} \mathrm{R}_{2}-\mathrm{PMM}_{\mathrm{R}}$ expressed in the local coordinate system $\left\{x_{i} y_{i} z_{i}\right\}$ is given by

$$
\begin{aligned}
& \mathbf{S}_{i r}=\left[\begin{array}{llllll}
1 & 0 & 0 & 0 & 0 & -d_{1}
\end{array}\right]^{\mathrm{T}} \\
& \mathbf{S}_{i 3}=\left[\begin{array}{lllllll}
l_{3} & m_{3} & n_{3} & d_{1} n_{3} & r_{b} n_{3}-r_{b} m_{3}-d_{1} l_{3}
\end{array}\right]^{\mathrm{T}} \\
& \left\{\mathbf{S}_{i}\right\}=\left\{\mathbf{S}_{i 4}=\left[\begin{array}{llllll}
l_{4} & m_{4} & n_{4} & y_{4} n_{4}-z_{4} m_{4} & z_{4} l_{4}-x_{4} n_{4} & x_{4} m_{4}-y_{4} l_{4}
\end{array}\right]^{\mathrm{T}}\right. \\
& \mathbf{S}_{i 5}=\left[\begin{array}{llllll}
0 & 0 & 0 & l_{5} & m_{5} & n_{5}
\end{array}\right]^{\mathrm{T}} \\
& \mathbf{S}_{i 6}=\left[\begin{array}{llllllll}
l_{4} & m_{4} & n_{4} & y_{6} n_{4}-z_{6} m_{4} & z_{6} l_{4}-x_{6} n_{4} & x_{6} m_{4}-y_{6} l_{4}
\end{array}\right]^{\mathrm{T}}
\end{aligned}
$$

This is a 5-system and a constraint screw can be calculated in terms of reciprocal screws, that is

$$
\left\{\mathbf{S}_{i}^{r}\right\}=\mathbf{S}_{i 2}^{r}=\left[\begin{array}{llllll}
l_{4} & m_{4} & n_{4} & d_{1} n_{4} & r_{b} n_{4}-r_{b} m_{4}-d_{1} l_{4}
\end{array}\right]^{\mathrm{T}}
$$

where $\left[\begin{array}{lll}-r_{b} & d_{1} & 0\end{array}\right]^{\mathrm{T}}$ is the position vectors of point $O$ expressed in local coordinate system $\left\{x_{i} y_{i} z_{i}\right\}$.

The constraint screw in Eq. (13) presents a constraint force which is parallel to screws $\mathbf{S}_{i 4}$ and $\mathbf{S}_{i 6}$ and passing the common point $O$. 
Hence, the varying constraints delivered by the $R_{1} P R_{1} R_{2}-P M M$ reconfigurable limb in different configurations are as the results of the phase change of the PMM subchain and the three limb configurations are distinguished by the condition of constraint free, one constraint couple and one constraint force respectively.

\section{Mobility Variation of the $3 R_{1} P_{1} R_{2}-P M M$ Metamorphic Parallel Mechanism}

The platform of the $3 R_{1} P R_{1} R_{2}-P M M$ parallel mechanism is connected to the base through three $R_{1} P R_{1} R_{2}-P M M$ reconfigurable hybrid limbs in Fig. 11. When the PMM subchain in each limb changes its configuration to the R phase, the center points of joint $B_{i}(i=1,2,3)$ form an equilateral triangle $\triangle \mathrm{B}_{1} \mathrm{~B}_{2} \mathrm{~B}_{3}$ and a circle centered at point $O$ with a radius of $r_{b}$. The global coordinate system $\{X Y Z\}$ is attached to the base with its original point located at the center $O$, the $X$-axis is aligned with $O B_{1}, Y$-axis is perpendicular to $O B_{1}$ and in the base plane and $Z$-axis is defined following the right-hand rule.

\subsection{Mobility of the $3 R_{1} P R_{1} R_{2}-P M M_{S}$ Configuration}

When the metamorphic parallel mechanism is in the $3 R_{1} P_{1} R_{2}-P M M_{S}$ configuration in Fig. 11, all the PMM subchains are acting in the source phase and the three limbs exert no constraint on the platform. Thus, the platform of the parallel mechanism has 6 degrees of freedom in this configuration.

\subsection{Mobility of the $3 R_{1} P_{1} R_{2}-P M M_{P}$ Configuration}

When all the PMM subchains in the three reconfigurable hybrid limbs change their configurations to P phase in Fig. 9, the parallel mechanism evolves into $3 \mathrm{R}_{1} \mathrm{PR}_{1} \mathrm{R}_{2}-\mathrm{PMM}_{\mathrm{P}}$ configuration in Fig. 12. By rotating the global coordinate system through $\varphi_{i}=2(i-1) \pi / 3$ about $Z$ axis, shifting it along axes parallel to $X$ and $Y$ axis and attaching its origin at point $A_{1}, A_{2}$, and $A_{3}$ respectively, three local coordinate systems $\left\{x_{i} y_{i} z_{i}\right\}$ (i=1,2, and 3) are introduced. Considering the local coordinate systems in Fig. 9 and Fig. 12, the constraint couple supplied by each limb expressed with respect to the corresponding local system $\left\{x_{i} y_{i} z_{i}\right\}$ in Fig. 12 are derived in Eq. (11).

The constraint screws in the local system can be transformed to the global coordinate system by the following transformation $[37,38]$.

$$
\mathbf{S}_{i}^{r}=\left({ }_{i}^{0} \mathbf{T}\right)\left(\mathbf{S}_{i 1}^{r}\right)^{\mathrm{T}}=\left[\begin{array}{cc}
\mathbf{R}\left(\varphi_{i}\right) & 0 \\
\mathbf{A}_{i} \mathbf{R}\left(\varphi_{i}\right) & \mathbf{R}\left(\varphi_{i}\right)
\end{array}\right]\left(\mathbf{S}_{i 1}^{r}\right)^{\mathrm{T}}
$$

where 


$$
\mathbf{A}_{i}=\left[\begin{array}{ccc}
0 & -o_{i z} & o_{i y} \\
o_{i z} & 0 & -o_{i x} \\
-o_{i y} & o_{i x} & 0
\end{array}\right], \mathbf{R}\left(\varphi_{i}\right)=\left[\begin{array}{ccc}
\cos \varphi_{i} & -\sin \varphi_{i} & 0 \\
\sin \varphi_{i} & \cos \varphi_{i} & 0 \\
0 & 0 & 1
\end{array}\right]
$$

in which $\left[\begin{array}{lll}o_{i x} & o_{i y} & o_{i z}\end{array}\right]^{\mathrm{T}}$ are the position vector of the origin point $o_{i}$ of the local systems expressed in the global coordinate system.

Hence, the constraint screws delivered by the three limbs expressed in the global coordinate system are obtained as

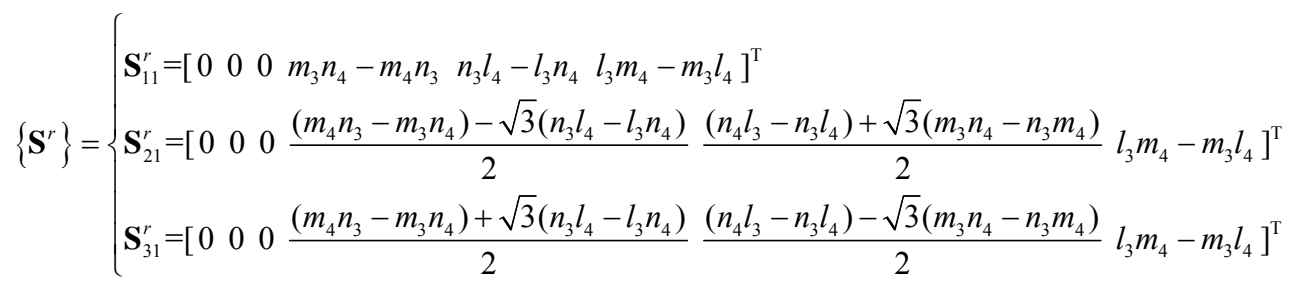

The constraint screws in Eq. (15) are three constraint couples in different directions, three rotational motions of the platform are constrained. It implies that the platform in this configuration has three degrees of freedom and implements three translational motions.

\subsection{Mobility of the $3 R_{1} P_{1} R_{2}-P_{M} M_{R}$ Configuration}

When all the PMM subchains change to $R$ phase, the parallel mechanism evolves into $3 R_{1} P R_{1} R_{2}-P_{M} M_{R}$ configuration in Fig. 13. The constraint exerted by each reconfigurable limb expressed with respect to the corresponding local system $\left\{x_{i} y_{i} z_{i}\right\}$ in Fig. 13 is obtained in Eq. (13) and the constraint screw system expressed in the global coordinate system is derived following Eq. (14), that is

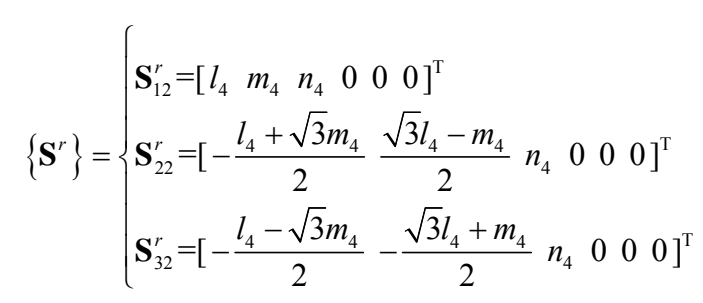

The constraint screws in Eq. (16) are three constraint forces intersecting at the common point $O$. Hence, the three translational motions are restricted and the platform has three degrees of freedom with rotational motions about point $O$, leading to a spherical parallel mechanism.

\subsection{Mobility of the $2 \mathbf{R}_{1} \mathbf{P R}_{1} \mathbf{R}_{2}-\mathbf{P M M}_{\mathrm{S}} / \mathbf{R}_{1} \mathbf{P R}_{\mathbf{1}} \mathbf{R}_{2}-\mathbf{P M M}_{\mathrm{P}}$ Configuration}

When the PMM subchain in one of the three limbs changes its configuration to P phase while the PMM subchains in the other two limbs remain in the source phase, the parallel mechanism reconfigure to $2 R_{1} P_{1} R_{2}-P_{M} M_{S} / R_{1} P R_{1} R_{2}-P M M_{P}$ configuration in 
Fig. 14 where the limb 1 changes to $R_{1} P_{1} R_{2}-P_{P M}$ configuration. There is no constraint exerted by limb 2 and limb 3 and the only constraint is the constraint couple exerted by limb 1 which is transformed to the global coordinate system following Eq. (14) and given by

$$
\left\{\mathbf{S}^{r}\right\}=\mathbf{S}_{11}^{r}=\left[\begin{array}{lllllll}
0 & 0 & 0 & m_{3} n_{4}-m_{4} n_{3} & n_{3} l_{4}-l_{3} n_{4} & l_{3} m_{4}-m_{3} l_{4}
\end{array}\right]^{\mathrm{T}}
$$

The constraint screw in Eq. (17) is a constraint couple, a rotational motion is constrained and the platform has five degrees of freedom with three translational motions and two rotational motions.

\subsection{Mobility of the $2 R_{1} P R_{1} R_{2}-P M M_{S} / R_{1} P R_{1} R_{2}-P_{M}$ Configuration}

When the PMM subchain in one of the three limbs changes its configuration to R phase while the PMM subchains in the other two limbs remain in source phase, the parallel mechanism evolves into $2 R_{1} P R_{1} R_{2}-P_{M} M_{S} / R_{1} P R_{1} R_{2}-P M M_{R}$ configuration in Fig. 15 where the limb 1 changes to $\mathrm{R}_{1} \mathrm{PR}_{1} \mathrm{R}_{2}-\mathrm{PMM}_{\mathrm{R}}$ configuration. The only constraint exerted to the platform is the constraint force by limb 1 and it is transformed to the global coordinate system following Eq. (14) and given by

$$
\left\{\mathbf{S}^{r}\right\}=\mathbf{S}_{12}^{r}=\left[\begin{array}{llllll}
l_{4} & m_{4} & n_{4} & 0 & 0 & 0
\end{array}\right]^{\mathrm{T}}
$$

The above constraint screw presents a constraint force passing point $O$ and pointing in direction $\left[\begin{array}{llll}l_{4} & m_{4} & n_{4}\end{array}\right]^{\mathrm{T}}$. Under such a condition, the translational motion in this direction is restricted and the platform has five degrees of freedom with three rotational motions and two translational motions.

\subsection{Mobility of the $R_{1} P R_{1} R_{2}-P M M_{S} / 2 R_{1} P R_{1} R_{2}-P M M_{P}$ Configuration}

When two PMM subchains in two limbs change their configurations to the P phase while the PMM subchain in the third limb remains in source phase, the parallel mechanism changes to $R_{1} P R_{1} R_{2}-P M M_{S} / 2 R_{1} P R_{1} R_{2}-P M M_{P}$ configuration in Fig. 16 in which the limb 1 and limb 2 are reconfigured. The constraint screws supplied by limb 1 and limb 2 are transformed to the global coordinate system following Eq. (14) and given by

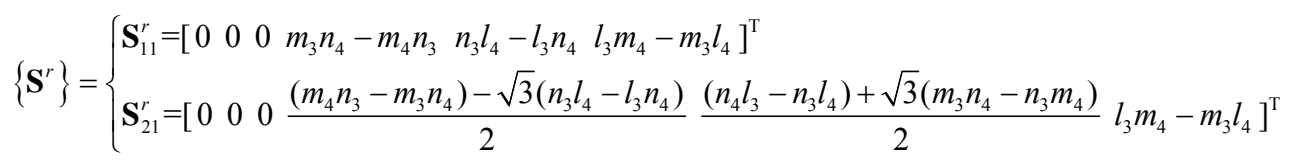

The constraint screws in Eq. (19) are two constraint couples in different directions leading to restriction of the rotational motions around these directions. Thus, the platform has four degrees of freedom with three translational motions and one rotational motion.

5.7 Mobility of the $\mathbf{R}_{1} \mathbf{P R}_{1} \mathbf{R}_{2}-\mathrm{PMM}_{\mathrm{S}} / 2 \mathrm{R}_{1} \mathbf{P R}_{1} \mathbf{R}_{2}-\mathrm{PMM}_{\mathrm{R}}$ Configuration 
When two PMM subchains in two limbs change their configurations to R phase while the PMM subchain in the third limb remains in the source phase, the parallel mechanism reconfigures to $R_{1} P R_{1} R_{2}-P_{M} M_{S} / 2 R_{1} P R_{1} R_{2}-P M M_{R}$ configuration in Fig. 17 in which limb 1 and limb 2 change to $R_{1} P_{1} R_{2}-P_{M}$ configuration. The constraints supplied by limb 1 and limb 2 are transformed to the global coordinate system following Eq. (14) and given by

$$
\left\{\mathbf{S}^{r}\right\}=\left\{\begin{array}{l}
\mathbf{S}_{12}^{r}=\left[\begin{array}{llllll}
l_{4} & m_{4} & n_{4} & 0 & 0 & 0
\end{array}\right]^{\mathrm{T}} \\
\mathbf{S}_{22}^{r}=\left[\begin{array}{llllll}
-\frac{l_{4}+\sqrt{3} m_{4}}{2} & \frac{\sqrt{3} l_{4}-m_{4}}{2} n_{4} & 0 & 0 & 0
\end{array}\right]^{\mathrm{T}}
\end{array}\right.
$$

The constraint screws in Eq. (20) represent two constraint forces in different directions and intersect at point $O$. Thus, the translational motions in these directions are constrained and the platform has four degrees of freedom with three rotational motions and one translational motion.

\subsection{Mobility of the $R_{1} P R_{1} R_{2}-P M M_{S} / R_{1} P R_{1} R_{2}-P M M_{R} / R_{1} P R_{1} R_{2}-P M M_{P}$ Configuration}

When the three reconfigurable limbs are in three different configurations respectively, the parallel mechanism changes to $\mathrm{R}_{1} \mathrm{PR}_{1} \mathrm{R}_{2}-\mathrm{PMM}_{\mathrm{S}} / \mathrm{R}_{1} \mathrm{PR}_{1} \mathrm{R}_{2}-\mathrm{PMM}_{\mathrm{R}} / \mathrm{R}_{1} \mathrm{PR}_{1} \mathrm{R}_{2}-\mathrm{PMM}_{\mathrm{P}}$ configuration as in Fig. 18 where limb 3 unchanged, limb 1 in $\mathrm{R}_{1} \mathrm{PR}_{1} \mathrm{R}_{2}-$ $\mathrm{PMM}_{\mathrm{P}}$ configuration and limb 2 in $\mathrm{R}_{1} \mathrm{PR}_{1} \mathrm{R}_{2}-\mathrm{PMM}_{\mathrm{R}}$ configuration. In this configuration, the constraints exerted to the platform by limb 1 and limb 2 are transformed to the global coordinate system following Eq. (14) and given by

$$
\left\{\mathbf{S}^{r}\right\}=\left\{\begin{array}{l}
\mathbf{S}_{11}^{r}=\left[\begin{array}{llllllll}
0 & 0 & 0 & m_{3} n_{4}-m_{4} n_{3} & n_{3} l_{4}-l_{3} n_{4} & l_{3} m_{4}-m_{3} l_{4}
\end{array}\right]^{\mathrm{T}} \\
\mathbf{S}_{22}^{r}=\left[\begin{array}{llllll}
-\frac{l_{4}+\sqrt{3} m_{4}}{2} & \frac{\sqrt{3} l_{4}-m_{4}}{2} n_{4} & 0 & 0 & 0
\end{array}\right]^{\mathrm{T}}
\end{array}\right.
$$

The constraint screw system in Eq. (21) contains one constraint force passing point $O$ and one constraint couple. Hence, a rotational motion and a translational motion are constrained and the platform has four degrees of freedom with two translational motions and two rotational motions.

\subsection{Mobility of the $2 R_{1} P R_{1} R_{2}-P_{M} M_{R} / R_{1} P R_{1} R_{2}-P_{M} M_{P}$ Configuration}

When the reconfigurable limbs 1 and 2 change to $2 R_{1} P R_{1} R_{2}-P M M_{R}$ configuration and the limb 3 changes to $R_{1} P R_{1} R_{2}-P_{M M}$ configuration, the parallel mechanism evolves into $2 R_{1} P R_{1} R_{2}-P M M_{R} / R_{1} P R_{1} R_{2}-P M M_{P}$ configuration in Fig. 19. The constraints provided by the three limbs are transformed to the global coordinate system following Eq. (14) and given by

$$
\left\{\mathbf{S}^{r}\right\}=\left\{\begin{array}{l}
\mathbf{S}_{12}^{r}=\left[\begin{array}{llllll}
l_{4} & m_{4} & n_{4} & 0 & 0 & 0
\end{array}\right]^{\mathrm{T}} \\
\mathbf{S}_{22}^{r}=\left[\begin{array}{llllll}
-\frac{l_{4}+\sqrt{3} m_{4}}{2} & \frac{\sqrt{3} l_{4}-m_{4}}{2} n_{4} & 0 & 0 & 0
\end{array}\right]^{\mathrm{T}} \\
\mathbf{S}_{31}^{r}=\left[\begin{array}{lllll}
0 & 0 & 0 & \frac{\left(m_{4} n_{3}-m_{3} n_{4}\right)+\sqrt{3}\left(n_{3} l_{4}-l_{3} n_{4}\right)}{2} \frac{\left(n_{4} l_{3}-n_{3} l_{4}\right)-\sqrt{3}\left(m_{3} n_{4}-n_{3} m_{4}\right)}{2} l_{3} m_{4}-m_{3} l_{4}
\end{array}\right]^{\mathrm{T}}
\end{array}\right.
$$


The constraint screw system in Eq. (22) is formed by two constraint forces passing the common point $O$ and one constraint couple. Thus, one rotational motion and two translational motions are constrained and the platform has three degrees of freedom with two rotational motions and one translational motion.

\subsection{Mobility of the $2 R_{1} P R_{1} R_{2}-P M M_{P} / R_{1} P R_{1} R_{2}-P M M_{R}$ Configuration}

When the reconfigurable limbs 1 and 2 change to $2 R_{1} P R_{1} R_{2}-P M M_{p}$ configuration and the limb 3 changes to $R_{1} P R_{1} R_{2}-P M M_{R}$ configuration, the parallel mechanism evolves into $2 R_{1} P R_{1} R_{2}-P M M_{P} / R_{1} P R_{1} R_{2}-P M M_{R}$ configuration in Fig. 20. The constraints provided by the three limbs are transformed to the global coordinate frame following Eq. (14) and given by

$$
\left\{\mathbf{S}^{r}\right\}=\left\{\begin{array}{l}
\mathbf{S}_{11}^{r}=\left[\begin{array}{llllll}
0 & 0 & 0 & m_{3} n_{4}-m_{4} n_{3} & n_{3} l_{4}-l_{3} n_{4} & l_{3} m_{4}-m_{3} l_{4}
\end{array}\right]^{\mathrm{T}} \\
\mathbf{S}_{21}^{r}=\left[\begin{array}{llll}
0 & 0 & 0 & \frac{\left(m_{4} n_{3}-m_{3} n_{4}\right)-\sqrt{3}\left(n_{3} l_{4}-l_{3} n_{4}\right)}{2} \frac{\left(n_{4} l_{3}-n_{3} l_{4}\right)+\sqrt{3}\left(m_{3} n_{4}-n_{3} m_{4}\right)}{2} l_{3} m_{4}-m_{3} l_{4}
\end{array}\right]^{\mathrm{T}} \\
\mathbf{S}_{32}^{r}=\left[\begin{array}{lllll}
-\frac{l_{4}-\sqrt{3} m_{4}}{2}-\frac{\sqrt{3} l_{4}+m_{4}}{2} n_{4} & 0 & 0 & 0
\end{array}\right]^{\mathrm{T}}
\end{array}\right.
$$

The constraint screws in Eq. (23) are two constraint couples and one constraint force. Hence, two rotational motions and one translational motion are restricted and the platform has three degrees of freedom with one rotational motion and two translational motions.

\section{Actuation Scheme}

As aforementioned in section 2, two motors mounted to joint $A$ and joint $D$ are necessary for reconfiguring working phases and implement certain motion in each phase of the PMM. Thus, six actuators have to be adopted for the three PMM subchains which are integrated in each metamorphic parallel mechanism in Table 1. Since the serial subchain integrated in each reconfigurable hybrid limb generates one constraint force and one constraint couple, the three reconfigurable limbs exert three constraint forces and three constraint couples which form a 6-system in a general configuration when the motors mounted to the PMM subchains are locked, It implies that this six motors mounted to the three PMM subchains are efficient to actuation validity conditions for parallel mechanisms [39]. In other words, the six actuators can sufficiently drive the configuration switch of the parallel mechanisms leading to mobility variation of the platform. All the motors are nearly mounted to the base, which will benefit the dynamic performances of this kind of metamorphic parallel mechanisms.

It is worth mentioning that the motor mounted to joint $D$ is resting while the PMM changes to $\mathrm{P}$ phase driven by the motor mounted to joint $A$. When the PMM changes to $\mathrm{R}$ phase, the only active motor is the one mounted to joint $D$ since the motor mounted to joint $A$ is locked by proper physical constraints. We can mount a brake system on joint $D$ to lock this joint in $\mathrm{P}$ phase. Therefore, with the metamorphic parallel mechanism in different mobility configurations, the number of working motors need to be controlled is equal to the mobility of the mechanism as listed in Table 2. 


\section{Singularity analysis}

Singular configurations appear both in serial mechanisms and parallel mechanisms. Generally, we can classify the singularities of a parallel mechanism into three types [40], i.e. inverse kinematic singularity, direct kinematic singularity and combined singularity. The third type of singularity is ignored here since it can be easily identified as long as the inverse kinematic singularities and direct kinematic singularities are determined. Next, inverse kinematic singularity and direct kinematic singularity analysis of the $3 \mathrm{R}_{1} \mathrm{PR}_{1} \mathrm{R}_{2}$-PMM metamorphic parallel mechanism will be conducted.

\subsection{Inverse kinematic singularity}

Inverse kinematic singularities usually occur at the workspace boundary with the twist system of a limb degenerated. In the $3 \mathrm{R}_{1} \mathrm{PR}_{1} \mathrm{R}_{2}-\mathrm{PMM}$ mechanism, there are three identical $\mathrm{R}_{1} \mathrm{PR}_{1} \mathrm{R}_{2}-\mathrm{PMM}$ hybrid limbs, this kind if limb will be inverse singular in the following conditions.

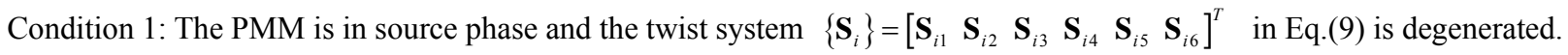

Example: The hybrid limb in $R_{1} \mathrm{PR}_{1} \mathrm{R}_{2}-\mathrm{PMM}_{\mathrm{P}}$ and $\mathrm{R}_{1} \mathrm{PR}_{1} \mathrm{R}_{2}-\mathrm{PMM}_{\mathrm{R}}$ configurations can be regarded as inverse kinematic singularities of the limb in $\mathrm{R}_{1} \mathrm{PR}_{1} \mathrm{R}_{2}-\mathrm{PMM}_{\mathrm{S}}$ configuration. However, these two kinds of singularities are employed to realize the reconfiguration of the hybrid limb and parallel mechanism.

Condition 2: The PMM is in P phase and the twist system $\left\{\mathbf{S}_{i}\right\}=\left[\begin{array}{lllll}\mathbf{S}_{i p} & \mathbf{S}_{i 3} & \mathbf{S}_{i 4} & \mathbf{S}_{i 5} & \mathbf{S}_{i 6}\end{array}\right]^{T}$ in Eq.(10) is degenerated.

Example: When the limb moves to the configuration with the prismatic joint in the serial subchain reaching its minimum stroke, as in Fig.21(a), twist system $\left\{\mathbf{S}_{i}\right\}$ degenerates into a 4-system.

Condition 3: The PMM is in R phase and the twist system $\left\{\mathbf{S}_{i}\right\}=\left[\begin{array}{lllll}\mathbf{S}_{i r} & \mathbf{S}_{i 3} & \mathbf{S}_{i 4} & \mathbf{S}_{i 5} & \mathbf{S}_{i 6}\end{array}\right]^{T}$ in Eq.(12) is degenerated.

Example: when the prismatic joint in the PMM reaches its maximal stroke as in Fig.21(b), twist system $\left\{\mathbf{S}_{i}\right\}$ is degenerated and the hybrid limb is inverse singular.

\subsection{Direct kinematic singularity}

In direct singular configurations, the output link of a mechanism can have infinitesimal motion in some directions and the mechanism is out of control. To begin with, direct singularities of the PMM should be concerned. It is easy to find that the PMM is direct kinematic singular only when all the links are coincident because of the special dimensional parameters.

When the PMM is not in direct singular configuration, all the link of the PMM will be immobile with the actuated joins locked, as a result, the hybrid limb $i(i=1,2,3)$ is equivalent to its inner 4-DOF serial subchain, and will exert a couple wrench $\mathbf{S}_{i C}^{r}$ and a force wrench $\mathbf{S}_{i F}^{r}$ on the moving platform, in which $\mathbf{S}_{i C}^{r}$ is perpendicular to the axes of joint $F_{i}$ and $G_{i}, \mathbf{S}_{i F}^{r}$ is parallel to the axis of joint $G_{i}$ and intersect with the axis of joint $F_{i}$, as in Fig.22. The couple and force wrenches generated by all the three limbs of 
the reconfigurable parallel mechanism will form a system $\mathbf{S}^{r}=\left[\begin{array}{lllllll}\mathbf{S}_{1 C}^{r} & \mathbf{S}_{1 F}^{r} & \mathbf{S}_{2 C}^{r} & \mathbf{S}_{2 F}^{r} & \mathbf{S}_{3 C}^{r} & \mathbf{S}_{3 F}^{r}\end{array}\right]^{T}$. In general conditions, $\mathbf{S}^{r}$ is a 6-system and will restrict all the six DOFs of the moving platform. However, when the six wrenches in $\mathbf{S}^{r}$ are dependent, rank of $\mathbf{S}^{r}$ will be less than 6 and the whole mechanism will be out of control, which means, direct singularities are appeared. Therefore, we can determine the direct singular configurations by checking whether the rank of $\mathbf{S}^{r}$ is 6 or less than 6 .

\section{Application Case Discussion}

The proposed metamorphic parallel mechanisms have the ability to achieve high operation speed and good dynamic performance because all the motors are either on (motors mounted to joints $A_{i}, i=1,2,3$ ) or close to (motors mounted to joints $D_{i}$, $i=1,2,3)$ the fixed base. Planar five-bar linkages are a kind of parallel mechanisms and have large stiffness [41-43], which have been integrated in hybrid limbs to construct parallel mechanisms with high stiffness and high resolution [44-46]. The three reconfigurable limbs in each metamorphic mechanism are arranged in parallel style and there exists a planar five-bar linkage acting as closed-loop subchain in each reconfigurable limb. These determine that the proposed metamorphic parallel mechanisms will have high stiffness performance, which can be further improved by performing optimization in several other respects such as the size and material of the links, mechanical transmission mechanisms, actuators and control system [47].

In view of the kinematic structure, the presented metamorphic parallel mechanisms consist of hybrid limbs in each of which a planar metamorphic mechanism (PMM) is employed as closed-loop subchain. The hybrid limbs of the metamorphic parallel mechanisms lead more joints comparing with conventional PKM designs consisting of serial kinematic limbs. Generally, a PKM design with serial kinematic limbs has certain degrees of freedom (DOFs) and motion characteristics and has unique functionality. In contrast, the proposed hybrid limbs are capable of reconfiguring their structures to distinct mobility configurations and the metamorphic parallel mechanisms with reconfigurable hybrid limbs are able to change their kinematic structure. The structure reconfiguration of the metamorphic parallel mechanism induced by the limb reconfiguration leads to adaptability of the mechanism for multifunction tasks. The proposed metamorphic parallel mechanisms can reconfigure their structures to ten distinct mobility configurations including change from a full 6-DOF mobility configuration to a spatial 3-DOF configuration without disassemble and reassemble of the mechanism. Each metamorphic parallel mechanism can be taken as the combination of ten parallel mechanism structures and used as multifunctional devices in applications with various task requirements. It takes much more effort and costs more resources to complete various tasks by employing more than one individual parallel structure which can has only one unchangeable mobility configuration.

Considering the adaptability and reconfigurability of the proposed metamorphic parallel mechanism, one of the promising application cases is to develop the multi-functional system for hybrid additive and subtractive manufacturing process. The additive manufacturing (usually called 3D printing) can only build rough model due to the diameter limitation of the nozzle, stair-stepping 
effect will occur on some sloped and freeform surfaces $[48,49]$. It is necessary to conduct finish machining on the rough model to get qualified product. In order to reduce the equipment cost and avoid second fixation, it is desired to design a machine tool that can integrate addictive manufacturing with subtractive manufacturing processes. Pure translational DOFs are required in the addictive manufacturing, while rotational DOFs are necessary to allow the tool working on some sloped and freeform surfaces in the subtractive manufacturing. According to these various tasks and multifunctional requirements, the proposed metamorphic parallel mechanisms are suitable in this application. As shown in Fig.23, the metamorphic mechanism works in configuration with pure translational DOFs to achieve high operation speed in the additive manufacturing stage. In this configuration, brake systems can be employed to lock the prismatic joints in the PMMs. When the rough model is built, the mechanism reconfigures to 3T2R configuration with assembled machining tool. Actuated by five motors, the mechanism can machine the rough model into expected final shape. The metamorphic parallel mechanism is able to implement multiple functions which require multiple conventional machines to complete the similar tasks. It implies multiple control systems have to be developed for different conventional machines, which cost more comparing to a unified control system of reconfigurable parallel mechanism.

The hybrid structure of kinematic limbs employed for constructing metamorphic parallel mechanisms has the merit of reconfigurability, while the large number of revolute and prismatic joints included in each of the metamorphic parallel mechanism lead to intricate design procedure and modeling of the system. In particular, linear actuators have to be purpose built and link dimensions should be properly optimized to realize the configuration change and to achieve reasonable workspaces. Manufacturing clearances of joints may reduce the accuracy of end-effector and complicated tasks in calibration of real devices. These aspects for industrial realization of the proposed mechanisms will be considered and addressed in the future works.

\section{Conclusions}

This paper presented a planar metamorphic mechanism (PMM) which was integrated in the construction of a new family of metamorphic parallel mechanisms. The PMM has three distinct working phases including the source phase, planar four bar parallelogram phase (P phase) and crank-slider phase ( $\mathrm{R}$ phase). The metamorphic parallel mechanisms were constructed with a platform, a base and three reconfigurable hybrid limbs. Each of the hybrid limbs consists of a serial subchain capable of supplying one constraint force and one constraint couple and a PMM subchain whose three working phases lead to three different configurations of the hybrid limb. The equivalent motion screws corresponding to each phase of the PMM and the constraints exerted by the reconfigurable hybrid limb in different configurations were identified based on screw theory. It revealed that the reconfigurable hybrid limb supplies one constraint force when the PMM subchain is in R phase and one constraint couple while

the PMM subchain changes to its $\mathrm{P}$ phase. The metamorphic parallel mechanisms are capable of evolving their mobility configurations from full 6-DOF to 5-, 4- and 3-DOF resorting to the configuration changes of the reconfigurable limbs. The actuation scheme reveals that six motors mounted to the three PMMs can actuate the metamorphic parallel mechanism and 
reconfigure mobility configurations with different kinds of motion. The proposed metamorphic parallel mechanisms can be used in many applications such as in hybrid additive and subtractive manufacturing process and painting robot that working on different kinds of surfaces.

\section{Acknowledgments}

The authors gratefully acknowledge the financial support of National Science Foundation of China under grant No.51075025, No.51175029 and No.51205016, Beijing Natural Science Foundation under grant No.3132019 and the Program for New Century Excellent Talents in University of China under grant No. NCET-12-0769.

\section{References}

[1] Wohlhart K. Kinematotropic Linkages. Recent Advances in Robot Kinematics. Berlin: Springer, 1996. $359-368$.

[2] Galletti C, Fanghella P. Single-loop kinematotropic mechanisms. Mech Mach Theor, 2001, 36(6): $743-761$.

[3] Zhang, L.P., Wang, D.L., Dai, J.S., 2008, "Biological Modeling and Evolution Based Synthesis of Metamorphic Mechanisms," Trans. ASME J. Mech. Des., 130(7), p.072303.

[4] Zhang, L.P., and Dai, J.S., 2009, "Reconfiguration of Spatial Metamorphic Mechanisms,” Trans. ASME J. Mech. Rob., 1(2), p. 011012.

[5] Dai, J. S., and Rees Jones, J., 1998, “Mobility in Metamorphic Mechanisms of Foldable/Erectable Kinds,” Proceedings 25th ASME Biennial Mechanisms and Robotics Conference, Atlanta, GA, Sept.

[6] Parise, J. J., Howell, L. L., and Magleby, S. P., 2000, “Ortho-Planar Mechanisms,"Proceedings of the 26th Biennial Mechanisms and Robotics Conference,Baltimore, MD, Paper No. DETC2000/MECH-14193.

[7] Chen I, Li H S, Cathala A. Mechatronic Design and Locomotion of Amoebot-A Metamorphic Underwater Vehicle[J]. Journal of Robotic Systems, 2003, 20(6): 307-314.

[8] Liu, C., and Yang, T., 2004, "Essence and Characteristics of Metamorphic Mechanisms and Their Metamorphic Ways," Proceedings 11th World Congress in Mechanism and Machine Science, Tianjing, China, Apr., pp. 1285-1288.

[9] Dai, J. S., and Rees Jones, J., 2005, “Matrix Representation of Topological Changes in Metamorphic Mechanisms,” Trans. ASME J. Mech. Des., 127(7), pp. 837-840.

[10] Wang, D. L., and Dai, J. S., 2007, “Theoretical Foundation of Metamorphic Mechanism and Its Synthesis,” Chin. J. Mech. Eng., 43(8), pp. 32-42.

[11] Dai, J. S., and Wang, D., 2007, “Geometric Analysis and Synthesis of the Metamorphic Robotic Hand,” Trans. ASME J. Mech. Des., 129(11), pp. 1191-1197.

[12] Dai, J. S., Wang, D. L., and Cui, L., 2009, “Orientation and Workspace Analysisof the Multifingered Metamorphic Hand- 
Metahand,” IEEE Trans. Robot., 25(4), pp. 942-947.

[13] Li, S.J., and Dai, J. S., 2012, "Structure Synthesis of Single-Driven Metamorphic Mechanisms Based on the Augmented Assur Groups,” Trans. ASME J. Mech. Rob., 4(3), p. 031004.

[14] Valsamos C, Moulianitis V, Aspragathos N. Index based optimal anatomy of a metamorphic manipulator for a given task[J]. Robotics and Computer-Integrated Manufacturing, 2012, 28(4): 517-529.

[15] Zhang, K.T., Emmanouil, E., Fang, Y.F., and Dai, J.S., 2012, “Type-Changeable Kinematic Pair Evolved Reconfigurable Parallel Mechanisms," Proceeding of the ASME/IFToMM International Conference on Reconfigurable Mechanisms and Robots (ReMAR 2012), Tianjin, China.

[16] Zhang, K., Dai, J. S., and Fang, Y., 2010, “Topology and Constraint Analysis of Phase Change in the Metamorphic Chain and Its Evolved Mechanism,” Trans. ASME J. Mech. Des., 132(12), p. 121001.

[17] Zhang, K.T., Dai, J.S., and Fang, Y.F., 2013, “Geometric Constraint and Mobility Variation of Two 3SPS Metamorphic Parallel Mechanisms,” Trans. ASME J. Mech. Des., 135(1), p. 011001.

[18] Gan, D., Dai, J. S., and Liao, Q., 2009, "Mobility Change in Two Types of Metamorphic Parallel Mechanisms," Trans. ASME J. Mech. Rob., 1(4), p. 041007.

[19] Gan, D. M., Dai, J. S., and Liao, Q. Z., 2010, “Constraint Analysis on Mobility Change in the Metamorphic Parallel Mechanism,” Mech. Mach. Theory, 45, pp.1864-1876.

[20] Gan, D. M., Dai, J. S., and Caldwell, D. G., 2011, "Constraint-Based Limb Synthesisand Mobility-Change-Aimed Mechanism Construction,” Trans. ASME,J. Mech. Des., 133(5), p. 051001.

[21] Gan D, Dai J S, Dias J, et al. Reconfigurability and unified kinematics modeling of a 3rTPS metamorphic parallel mechanism with perpendicular constraint screws. Robotics and Computer-Integrated Manufacturing, 2013(29): 121-128.

[22] Carbonari L, Callegari M, Palmieri G, et al. A new class of reconfigurable parallel kinematic machines[J]. Mechanism and Machine Theory, 2014, 79: 173-183.

[23] Palpacelli M C, Carbonari L, Palmieri G. Details on the Design of a Lockable Spherical Joint for Robotic Applications[J]. Journal of Intelligent \& Robotic Systems, 1-11.

[24] Palpacelli M C, Carbonari L, Palmieri G, et al. Analysis and Design of a Reconfigurable 3-DoF Parallel Manipulator for Multimodal Tasks[J]. IEEE/ASME Transactions on mechatronics, 2015.

[25] Carbonari L, Callegari M, Palmieri G, et al. Analysis of kinematics and reconfigurability of a spherical parallel manipulator[J]. Robotics, IEEE Transactions on, 2014, 30(6): 1541-1547.

[26] Fanghella P, Galletti C, Giannotti E. Parallel robots that change their group of motion[M]//Advances in Robot Kinematics. Springer Netherlands, 2006: 49-56. 
[27] Zlatanov D, Bonev I and Gosselin CM. Constraint singularities as configuration space singularities. In: Lenarcic J and Thomas F (eds) Advances in robot kinematics. Dordrecht: Kluwer Academic Press, 2002, pp.183-192.

[28] Gogu G. Branching singularities in kinematotropic parallel mechanisms. In: Computational kinematics. Berlin: Springer Berlin Heidelberg Press, 2009, pp.341-348.

[29] Li QC and Herve' JM. Parallel mechanisms with bifurcation of Schoenflies motion. IEEE Trans Rob 2009; 25: $158-164$.

[30] Ye W, Fang Y, Zhang K, et al. A new family of reconfigurable parallel mechanisms with diamond kinematotropic chain[J]. Mechanism and Machine Theory, 2014, 74: 1-9.

[31] Tsai, L. W., 1999, “The Enumeration of a Class of Three-DOF Parallel Manipulators," Proceedngs of the Tenth World Congress of the Theory of Machine and Mechanisms, Finland, Jun. 20-24, pp. 1121-1126.

[32] Liu, X. J., Wang, J., and Pritschow, G., 2005, “A New Family of Spatial 3-DoF Fully-Parallel Manipulators With High Rotational Capability,” Mech. Mach. Theory, 40(4), pp. 475-494.

[33] Tsai, L. W., 1999, Robot Analysis: The Mechanics of Serial and Parallel Manipulators, John Wiley, New York.

[34] Dai, J. S., and Rees, J. J., 2001, "Interrelationship Between Screw Systems and Corresponding Reciprocal Systems and Applications," Mech. Mach. Theory, 36(5), pp. 633-651.

[35] Fang, Y. F., and Tsai, L. W., 2004, "Enumeration of a Class of Overconstrained Mechanisms Using the Theory of Reciprocal Screws," Mech. Mach. Theory, 39(11), pp. 1175-1187.

[36] Zhang, K.T., Fang, Y.F., Fang, H.R., and Dai, J.S., 2010, “Geometry and Constraint Analysis of the three-special kinematic chain based parallel mechanism,” Trans. ASME J. Mech. Rob., 2(8), p. 031014.

[37] Dai, J. S., Huang, Z., and Lipkin, H., 2006, “Mobility of Overconstrained Parallel Mechanisms,” Trans. ASME J. Mech. Des., 128(1), pp. 220-229.

[38] Dai, J. S., 2012, “Finite Displacement Screw Operators with Embedded Chasles’ Motion,” Trans. ASME. J. Mech. Rob., 4(4), p. 041002 .

[39] Kong, X., and Gosselin, C. M., 2004, “Type Synthesis of 3-DOF Spherical Parallel Manipulators Based on Screw Theory,” Trans. ASME J. Mech. Des., 126(1), pp. 101-108.

[40] Tsai LW. Robot analysis: the mechanics of serial and parallel manipulators. John Wiley \& Sons, 1999.

[41] Asada H, Youcef-Toumi K. Analysis and design of a direct-drive arm with a five-bar-link parallel drive mechanism. Trans ASME; J Dyn Syst, Meas Control 1984;106(3):225 - 30.

[42] Kim J Y. Task based kinematic design of a two DOF manipulator with a parallelogram five-bar link mechanism[J]. Mechatronics, 2006, 16(6): 323-329. 
[43] Zhou H, Minupuri S, Indroju S, et al. Five-Bar Cylinder-Crank Two-DOF Parallel Manipulators for Singularity-Free Path Generation[C]//ASME 2009 International Mechanical Engineering Congress and Exposition. American Society of Mechanical Engineers, 2009: 41-48.

[44] Tsai L W, Tahmasebi F. Synthesis and analysis of a new class of six - degree - of - freedom parallel minimanipulators[J]. Journal of Robotic systems, 1993, 10(5): 561-580.

[45] Tahmasebi F, Tsai L W. Simplified and symmetrical five-bar linkage driver for manipulating a Six-Degree-of-Freedom Parallel" minimanipulator" with three inextensible limbs: U.S. Patent 5,301,566[P]. 1994-4-12.

[46] F. Tahmasebi, L.W. Tsai, Jacobian and stiffness analysis of a novel class of six-DOF parallel minimanipulators, ASME Des. Eng. Div. 47 (1992) $95-102$.

[47] Joshi S, Tsai L W. A comparison study of two 3-DOF parallel manipulators: One with three and the other with four supporting legs[J]. Robotics and Automation, IEEE Transactions on, 2003, 19(2): 200-209.

[48] Moylan S, Slotwinski J, Cooke A, Jurrens K, Donmez MA (2012) A review of test artifacts for additive manufacturing. NIST, available at 10.6028/NIST.IR.7858. Accessed Aug 2014

[49] S.O. Onuh, K.K.B. Hon, Optimising build parameters for improved surface finish in stereolithography, International Journal of Machine Tools and Manufacture, Volume 38, Issue 4, March 1998, Pages 329-342, ISSN 0890-6955, DOI: 10.1016/S0890-6955(97)00068-0. 


\section{Table Captions}

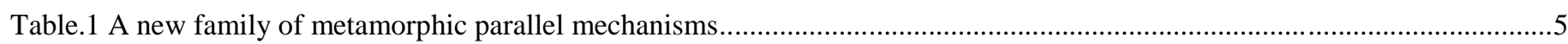

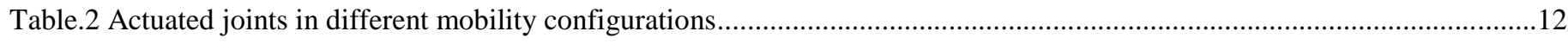


Table.1

\begin{tabular}{|c|c|c|}
\hline Limb type & \multicolumn{2}{|c|}{ Metamorphic Parallel Mechanisms } \\
\hline 4R-PMM & $3 \mathrm{R}_{1} \mathrm{R}_{1} \mathrm{R}_{1} \mathrm{R}_{2}-\mathrm{PMM}$ & $3 \mathrm{R}_{1} \mathrm{R}_{1} \mathrm{U}_{12}-\mathrm{PMM}$ \\
\hline \multirow{3}{*}{ 3R1P- PMM } & $3 \mathrm{PR}_{1} \mathrm{R}_{1} \mathrm{R}_{2}-\mathrm{PMM}$ & $3 \mathrm{PR}_{1} \mathrm{U}_{12}-\mathrm{PMM}$ \\
\hline & $3 \mathrm{R}_{1} \mathrm{PR}_{1} \mathrm{R}_{2}-\mathrm{PMM}$ & $3 \mathrm{R}_{1} \mathrm{PU}_{12}-\mathrm{PMM}$ \\
\hline & $3 \mathrm{R}_{1} \mathrm{R}_{1} \mathrm{PR}_{2}-\mathrm{PMM}$ & $3 \mathrm{R}_{1} \mathrm{R}_{1} \mathrm{C}_{2}-\mathrm{PMM}$ \\
\hline \multirow{4}{*}{ 2R2P- PMM } & $3 \mathrm{PPR}_{1} \mathrm{R}_{2}-\mathrm{PMM}$ & $3 \mathrm{PPU}_{12}-\mathrm{PMM}$ \\
\hline & $3 \mathrm{PPR}_{2} \mathrm{R}_{2}-\mathrm{PMM}$ & $3 \mathrm{PPU}_{22}-\mathrm{PMM}$ \\
\hline & $3 \mathrm{PR}_{1} \mathrm{PR}_{2}-\mathrm{PMM}$ & $3 \mathrm{PR}_{1} \mathrm{C}_{2}-\mathrm{PMM}$ \\
\hline & $3 \mathrm{R}_{1} \mathrm{PPR}_{2}-\mathrm{PMM}$ & $3 \mathrm{R}_{1} \mathrm{PC}_{2}-\mathrm{PMM}$ \\
\hline
\end{tabular}

Table.2

\begin{tabular}{c|l|l|l}
\hline \hline DoF & Configuration & Motions & Actuate joins \\
\hline 6 & $3 \mathrm{R}_{1} \mathrm{PR}_{1} \mathrm{R}_{2}-\mathrm{PMM}_{\mathrm{S}}$ & $3 \mathrm{~T} 3 \mathrm{R}$ & $A_{i}, D_{i}(i=1,2,3)$ \\
\hline \multirow{2}{*}{5} & $2 \mathrm{R}_{1} \mathrm{PR}_{1} \mathrm{R}_{2}-\mathrm{PMM}_{\mathrm{S}} / \mathrm{R}_{1} \mathrm{PR}_{1} \mathrm{R}_{2}-\mathrm{PMM}_{\mathrm{P}}$ & $3 \mathrm{~T} 2 \mathrm{R}$ & $A_{i}(i=1,2,3), D_{2}, D_{3}$ \\
\cline { 2 - 4 } & $2 \mathrm{R}_{1} \mathrm{PR}_{1} \mathrm{R}_{2}-\mathrm{PMM}_{\mathrm{S}} / \mathrm{R}_{1} \mathrm{PR}_{1} \mathrm{R}_{2}-\mathrm{PMM}_{\mathrm{R}}$ & $3 \mathrm{R} 2 \mathrm{~T}$ & $D_{i}(i=1,2,3), A_{2}, A_{3}$ \\
\hline \multirow{4}{*}{4} & $\mathrm{R}_{1} \mathrm{PR}_{1} \mathrm{R}_{2}-\mathrm{PMM}_{\mathrm{S}} / 2 \mathrm{R}_{1} \mathrm{PR}_{1} \mathrm{R}_{2}-\mathrm{PMM}_{\mathrm{P}}$ & $3 \mathrm{~T} 1 \mathrm{R}$ & $A_{i}(i=1,2,3), D_{3}$ \\
\cline { 2 - 4 } & $\mathrm{R}_{1} \mathrm{PR}_{1} \mathrm{R}_{2}-\mathrm{PMM}_{\mathrm{S}} / 2 \mathrm{R}_{1} \mathrm{PR}_{1} \mathrm{R}_{2}-\mathrm{PMM}_{\mathrm{R}}$ & $3 \mathrm{R} 1 \mathrm{~T}$ & $D_{i}(i=1,2,3), A_{3}$ \\
\cline { 2 - 4 } & $\mathrm{R}_{1} \mathrm{PR}_{1} \mathrm{R}_{2}-\mathrm{PMM}_{\mathrm{S}} / \mathrm{R}_{1} \mathrm{PR}_{1} \mathrm{R}_{2}-\mathrm{PMM}_{\mathrm{R}} / \mathrm{R}_{1} \mathrm{PR}_{1} \mathrm{R}_{2}-\mathrm{PMM}_{\mathrm{P}}$ & $2 \mathrm{R} 2 \mathrm{~T}$ & $A_{1}, A_{3}, D_{2}, D_{3}$ \\
\hline \multirow{5}{*}{3} & $3 \mathrm{R}_{1} \mathrm{PR}_{1} \mathrm{R}_{2}-\mathrm{PMM}_{\mathrm{R}}$ & $3 \mathrm{R}$ & $D_{i}(i=1,2,3)$ \\
\cline { 2 - 5 } & $2 \mathrm{R}_{1} \mathrm{PR}_{1} \mathrm{R}_{2}-\mathrm{PMM}_{\mathrm{R}} / \mathrm{R}_{1} \mathrm{PR}_{1} \mathrm{R}_{2}-\mathrm{PMM}_{\mathrm{P}}$ & $2 \mathrm{R} 1 \mathrm{~T}$ & $A_{3}, D_{1}, D_{2}$ \\
\cline { 2 - 5 } & $2 \mathrm{R}_{1} \mathrm{PR}_{1} \mathrm{R}_{2}-\mathrm{PMM}_{\mathrm{P}} / \mathrm{R}_{1} \mathrm{PR}_{1} \mathrm{R}_{2}-\mathrm{PMM}_{\mathrm{R}}$ & $2 \mathrm{~T} 1 \mathrm{R}$ & $A_{1}, A_{2}, D_{3}$ \\
\cline { 2 - 5 } & $3 \mathrm{R}_{1} \mathrm{PR}_{1} \mathrm{R}_{2}-\mathrm{PMM}_{\mathrm{P}}$ & $3 \mathrm{~T}$ & $A_{i}(i=1,2,3)$ \\
\hline \hline
\end{tabular}




\section{Figure Captions}

Fig.1 Two phases of the single driven planar metamorphic mechanism (a) planar four bar linkage phase; (b) crank-slider phase................................................. 3

Fig.2 Three phases of the PMM (a) source phase; (b) P phase; (c) R phase

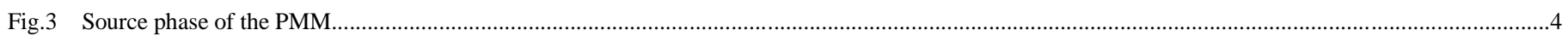

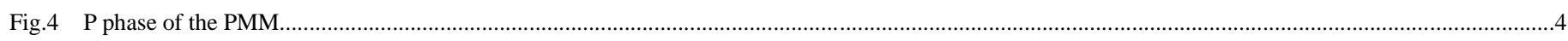

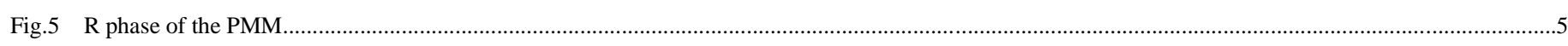

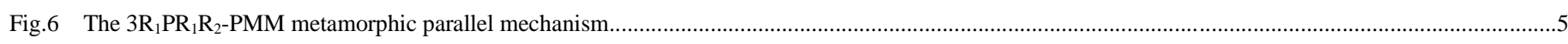

Fig.7 The $3 \mathrm{R}_{1} \mathrm{PR}_{1} \mathrm{R}_{2}$-PMM metamorphic parallel mechanism with all the PMMs in $\mathrm{R}$ phase

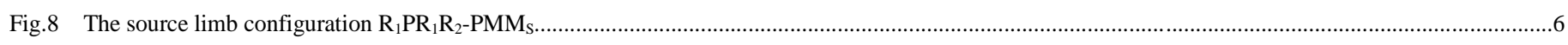

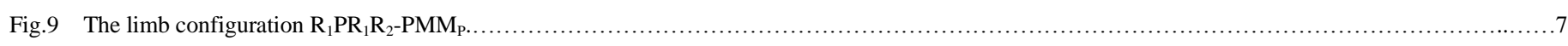

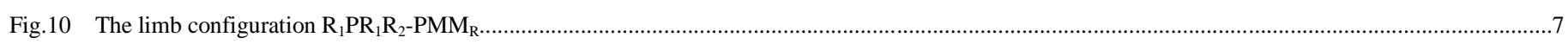

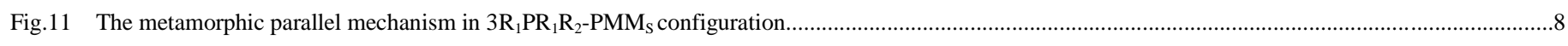

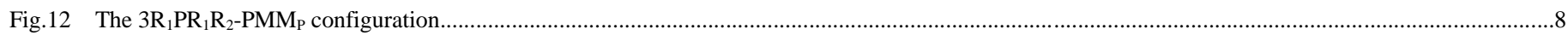

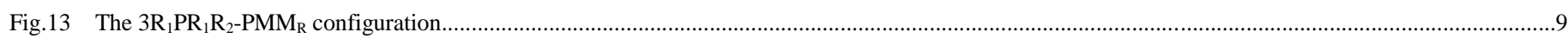

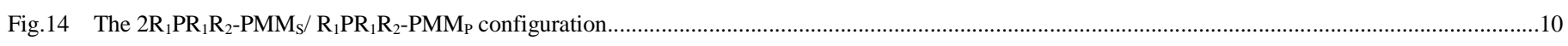

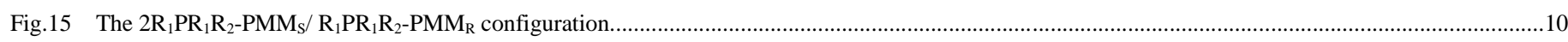

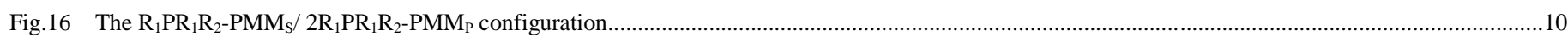

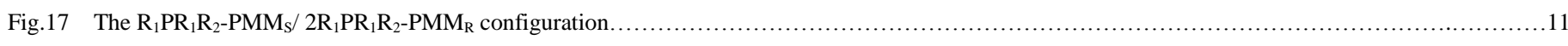

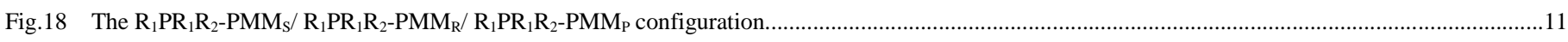

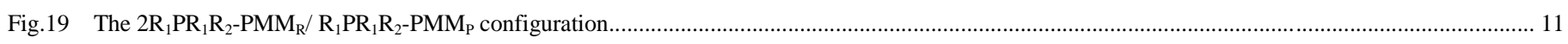

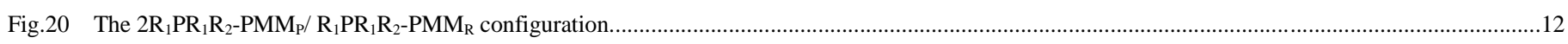

Fig.21 Inverse kinematic singularities: (a) in $\mathrm{R}_{1} \mathrm{PR}_{1} \mathrm{R}_{2}-\mathrm{PMM}_{\mathrm{P}}$ configuration, (b) in $\mathrm{R}_{1} \mathrm{PR}_{1} \mathrm{R}_{2}-\mathrm{PMM}_{\mathrm{R}}$ configuration........................................................13

Fig.22 Wrenches in the $3 \mathrm{R}_{1} \mathrm{PR}_{1} \mathrm{R}_{2}$-PMM metamorphic parallel mechanism......................................................................................................13

Fig.23 Hybrid additive and subtractive manufacturing process .....................................................................................................................................15 


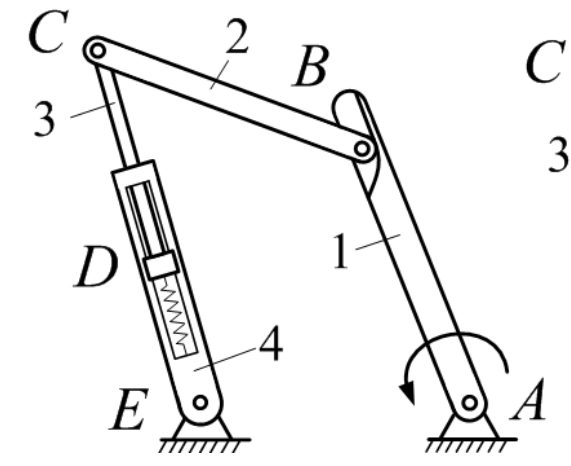

(a)

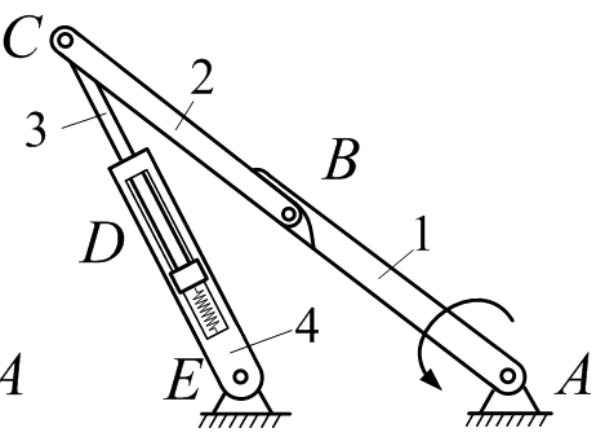

(b)

Fig. 1

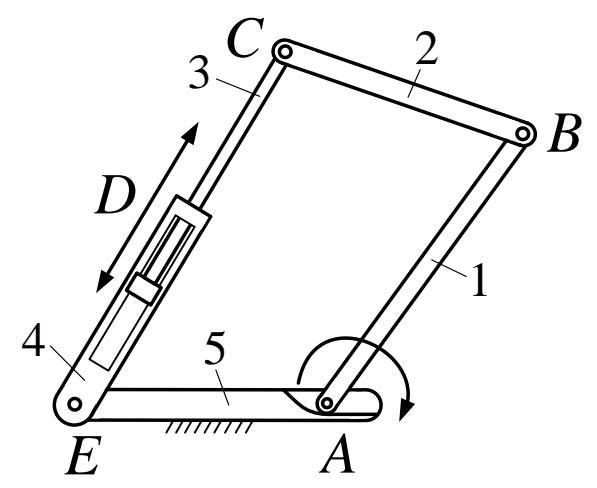

(a)

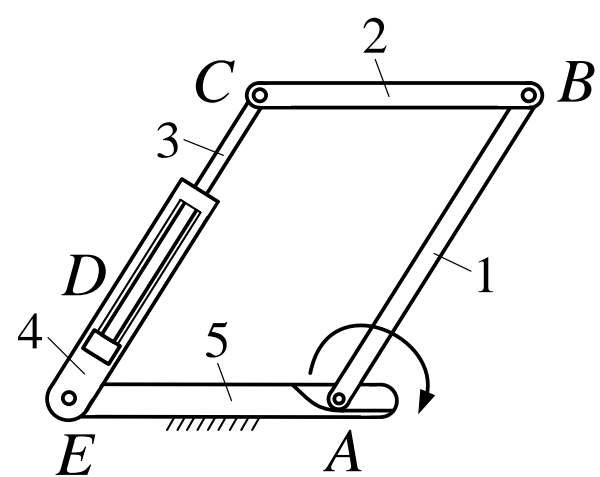

(b)

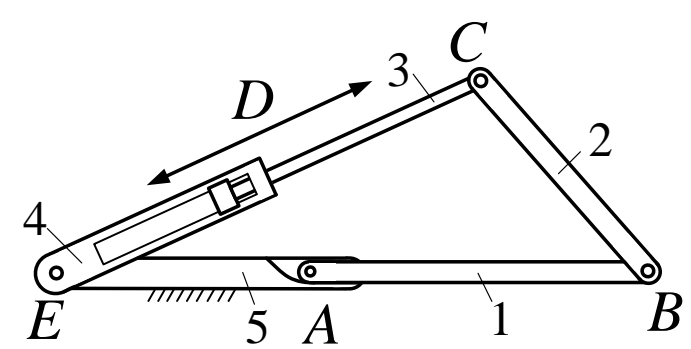

(c)

Fig. 2 


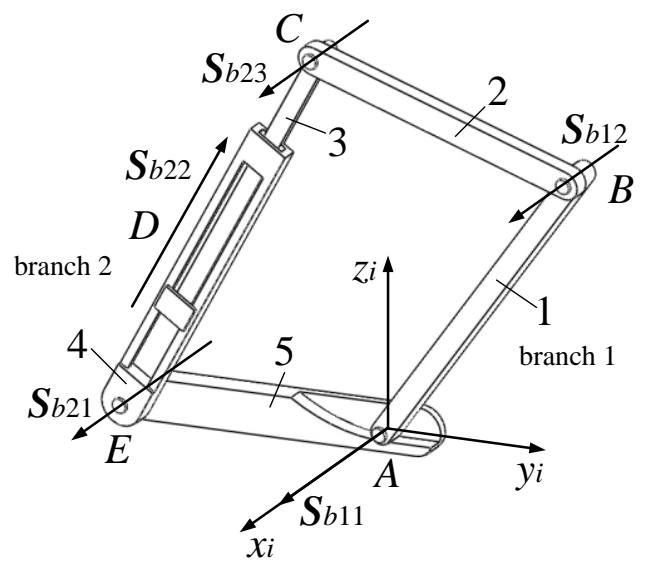

Fig. 3

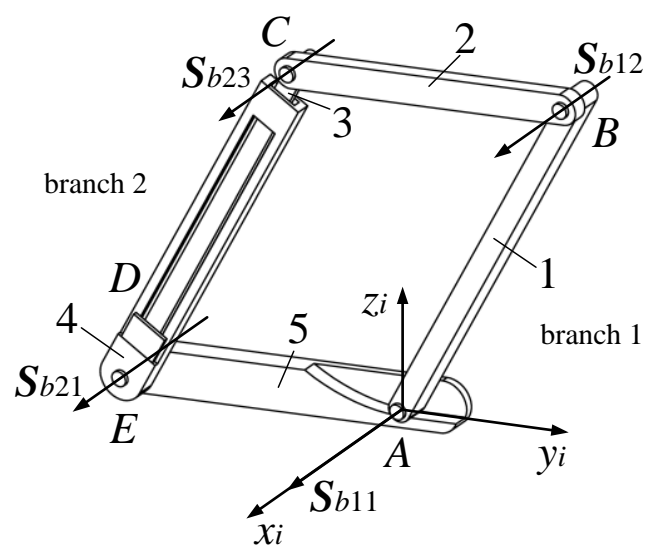

Fig. 4

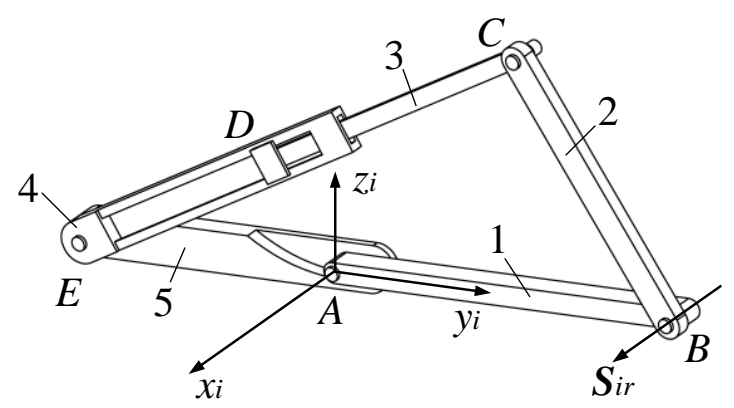

Fig. 5 


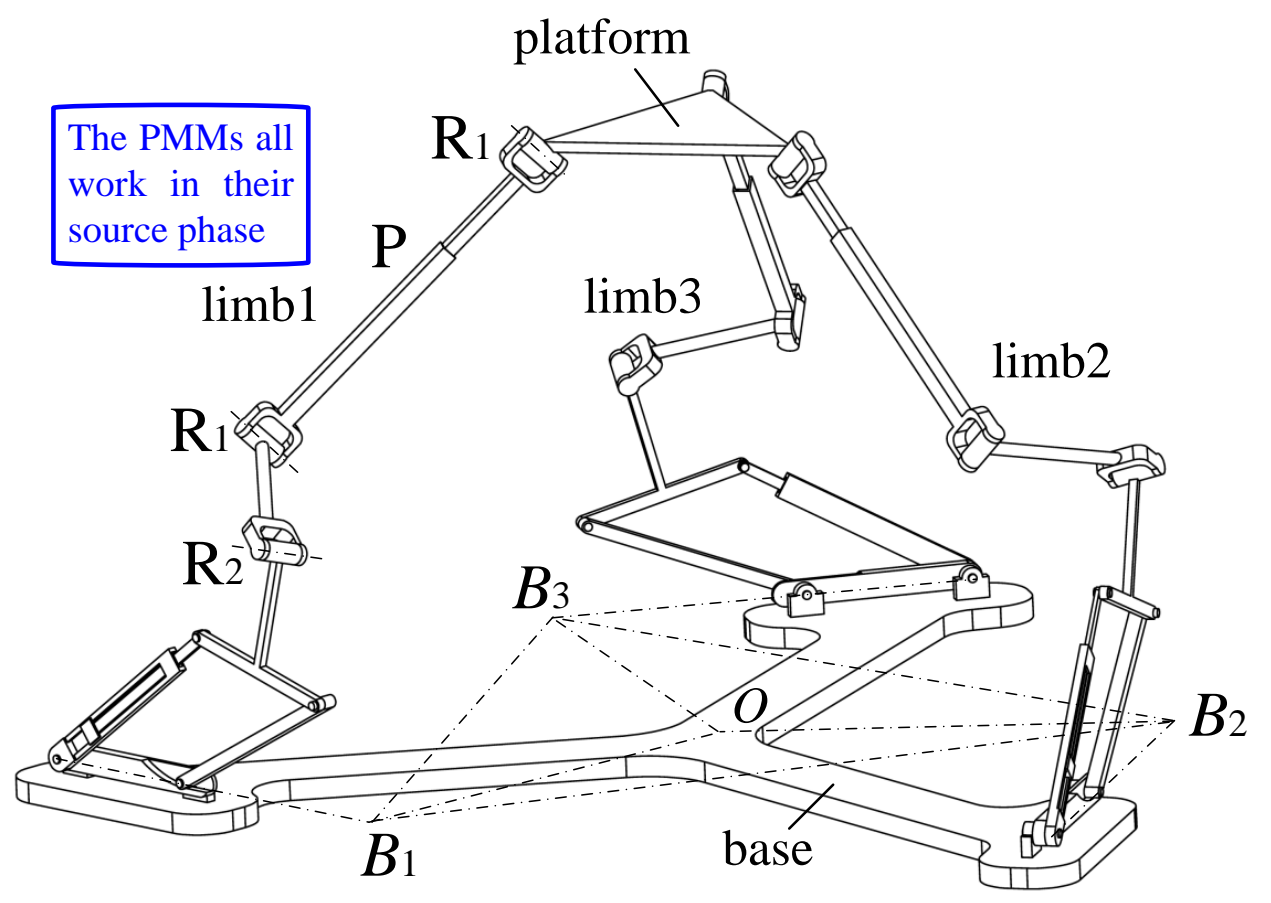

Fig. 6

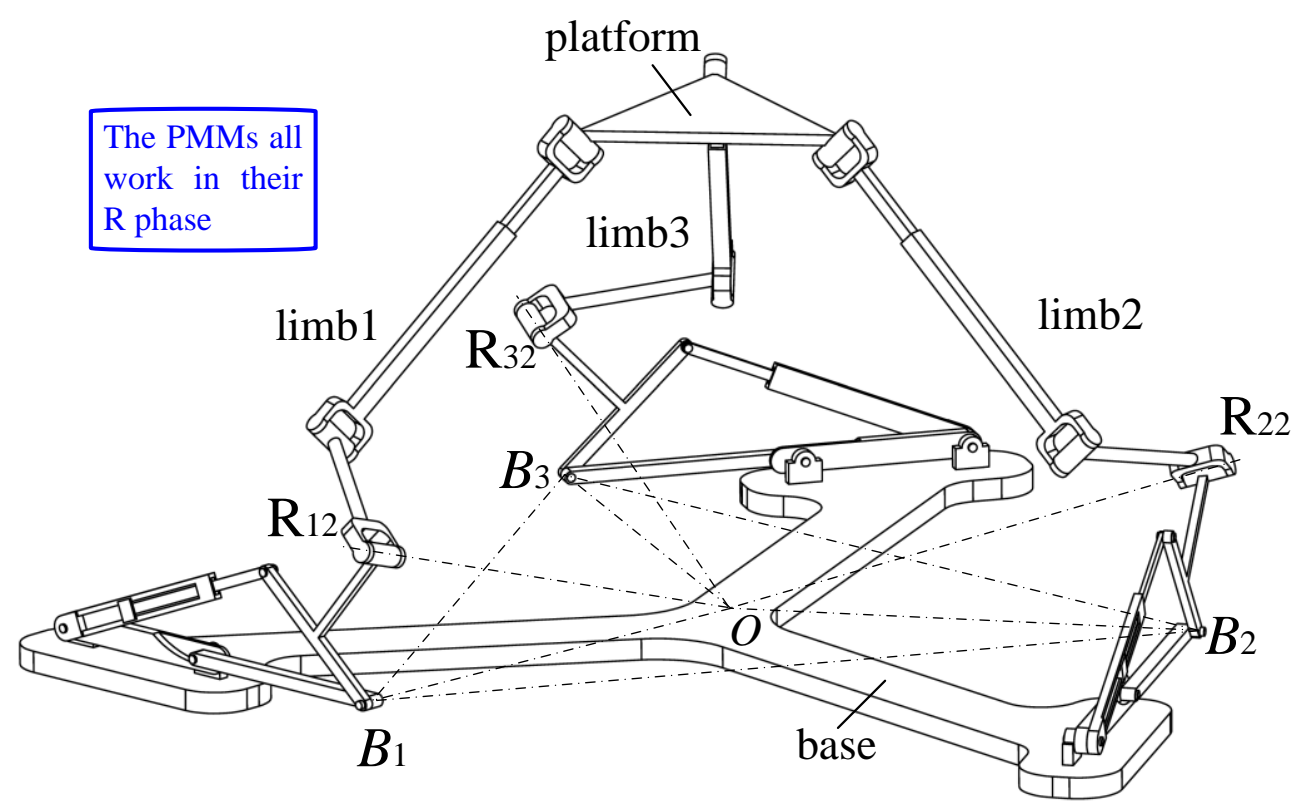

Fig. 7 


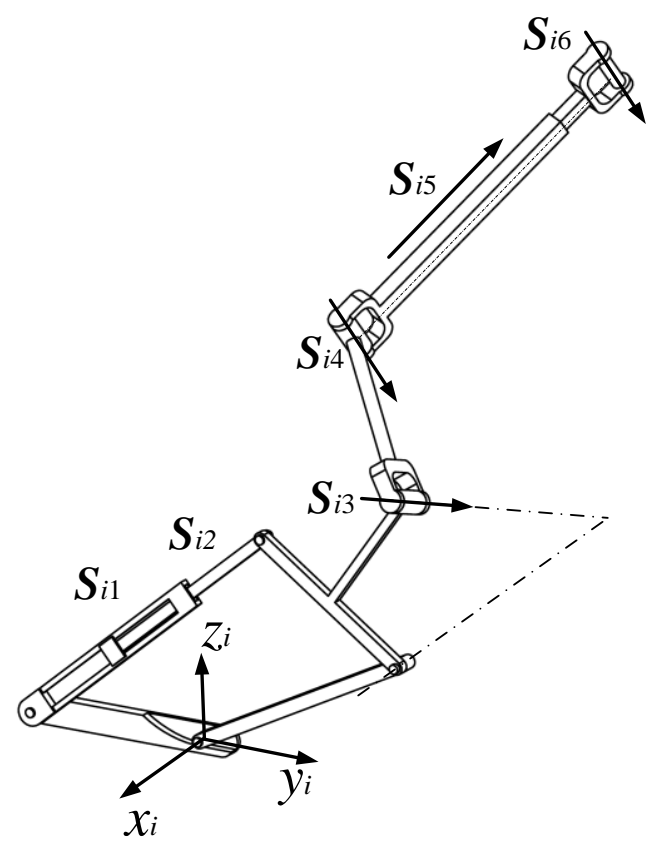

Fig. 8

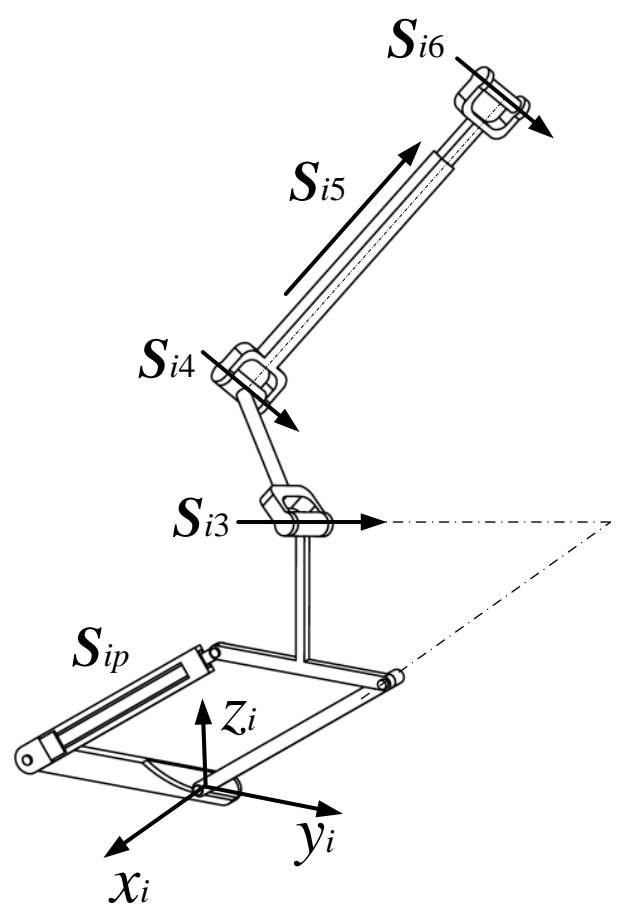

Fig. 9 


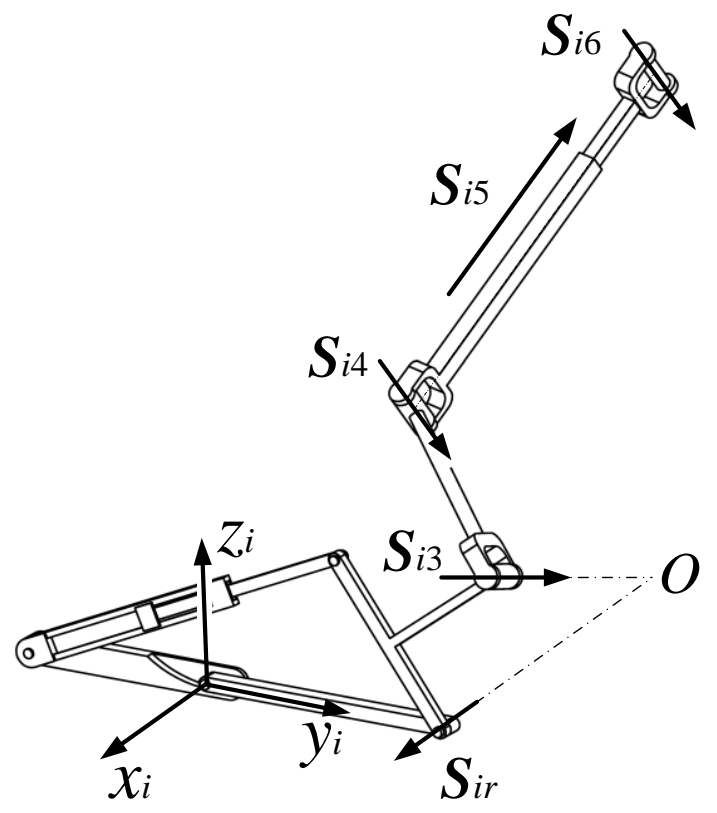

Fig. 10

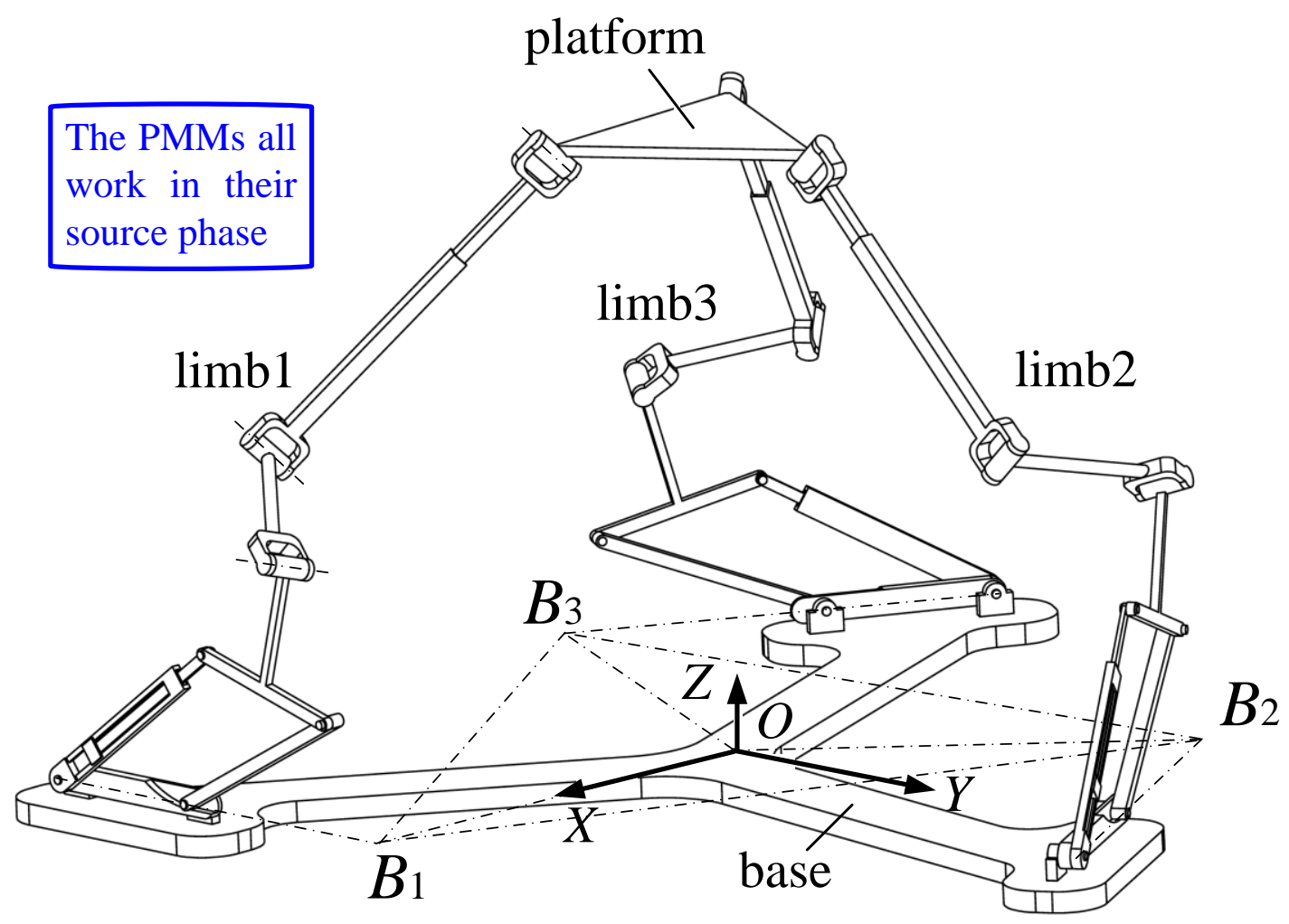

Fig.11 


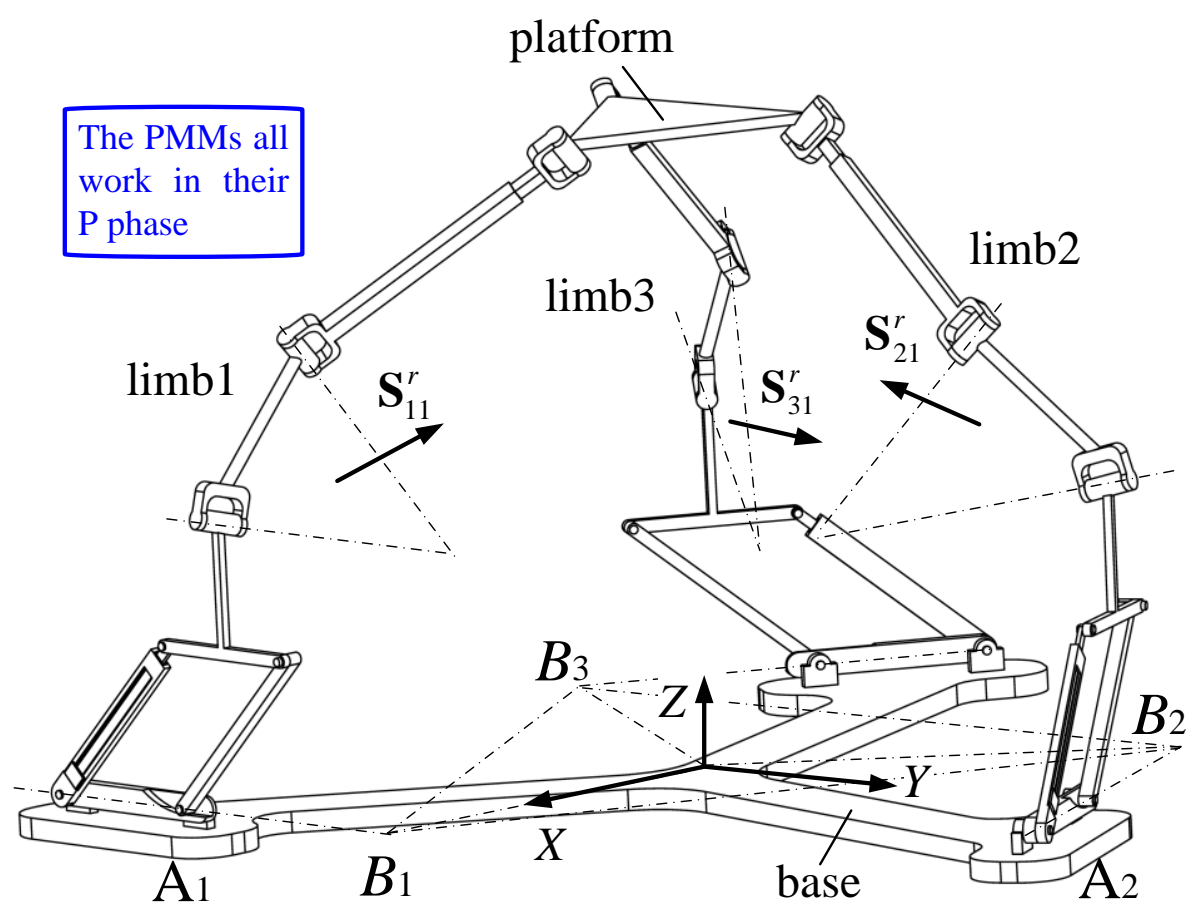

Fig.12

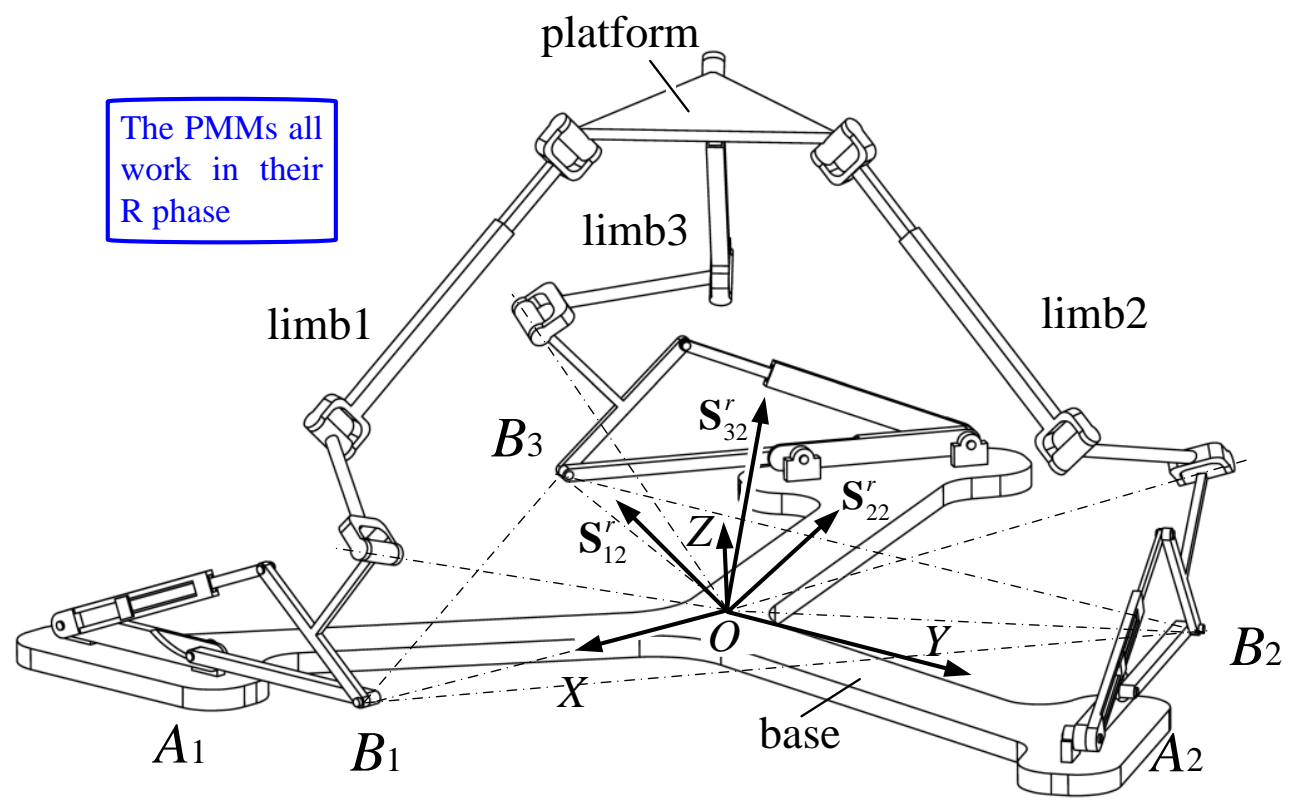

Fig.13 


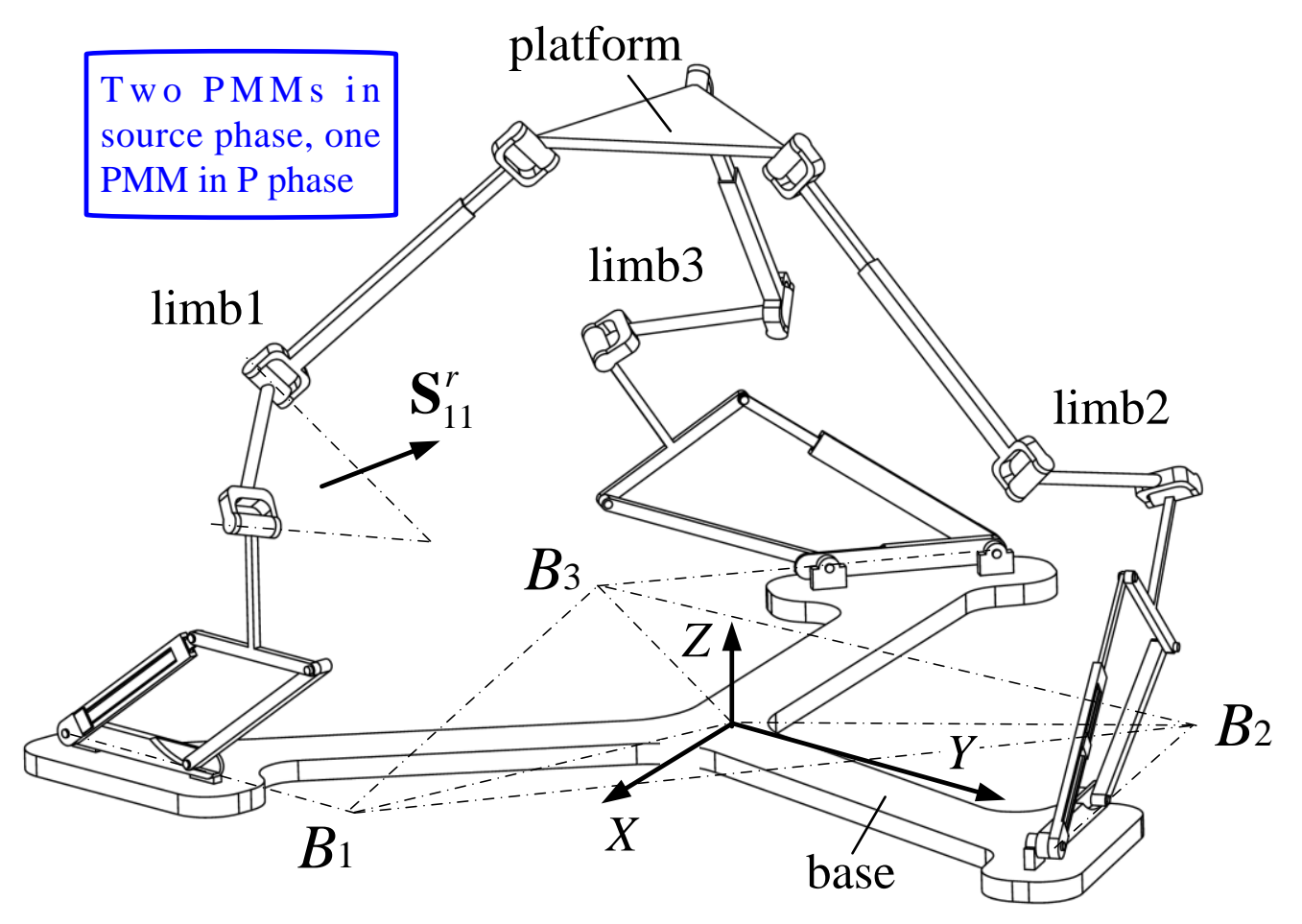

Fig.14

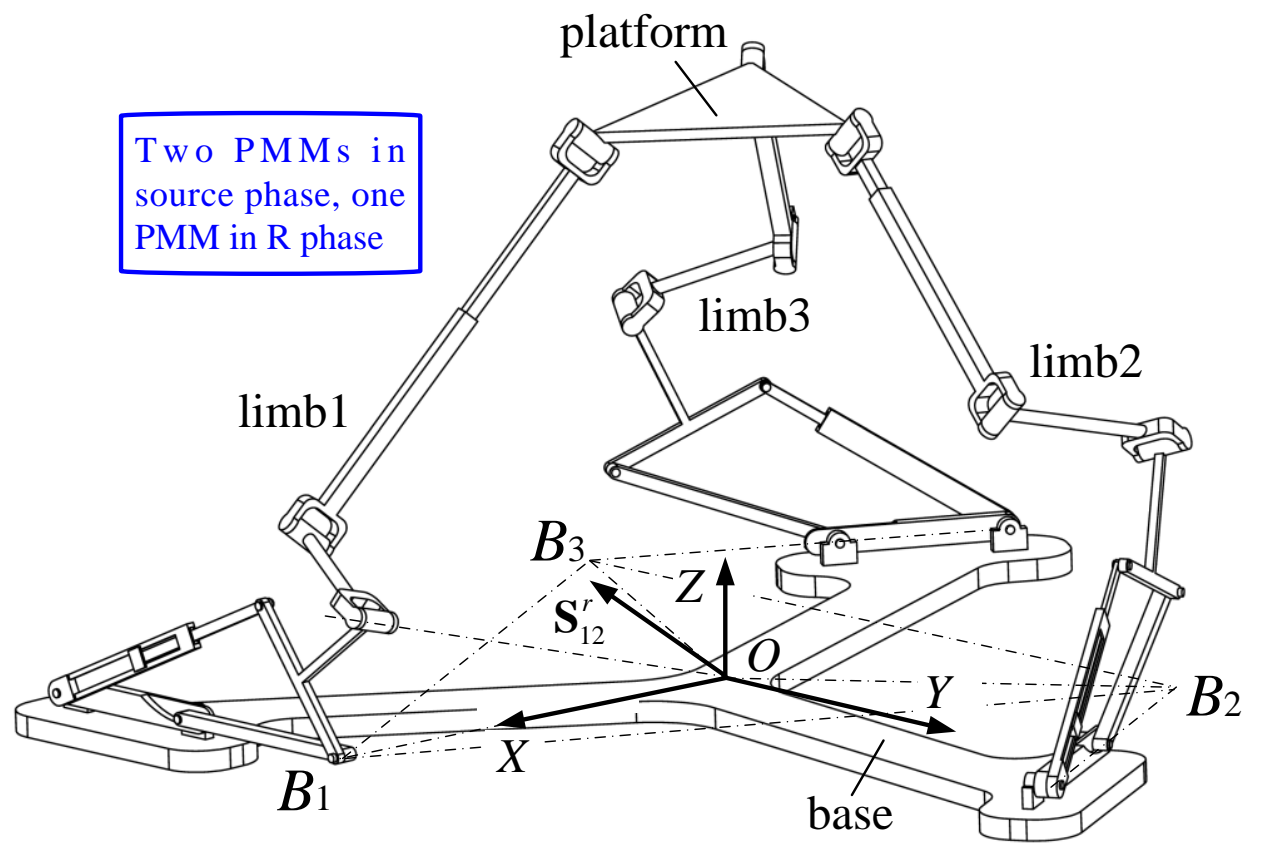

Fig.15 


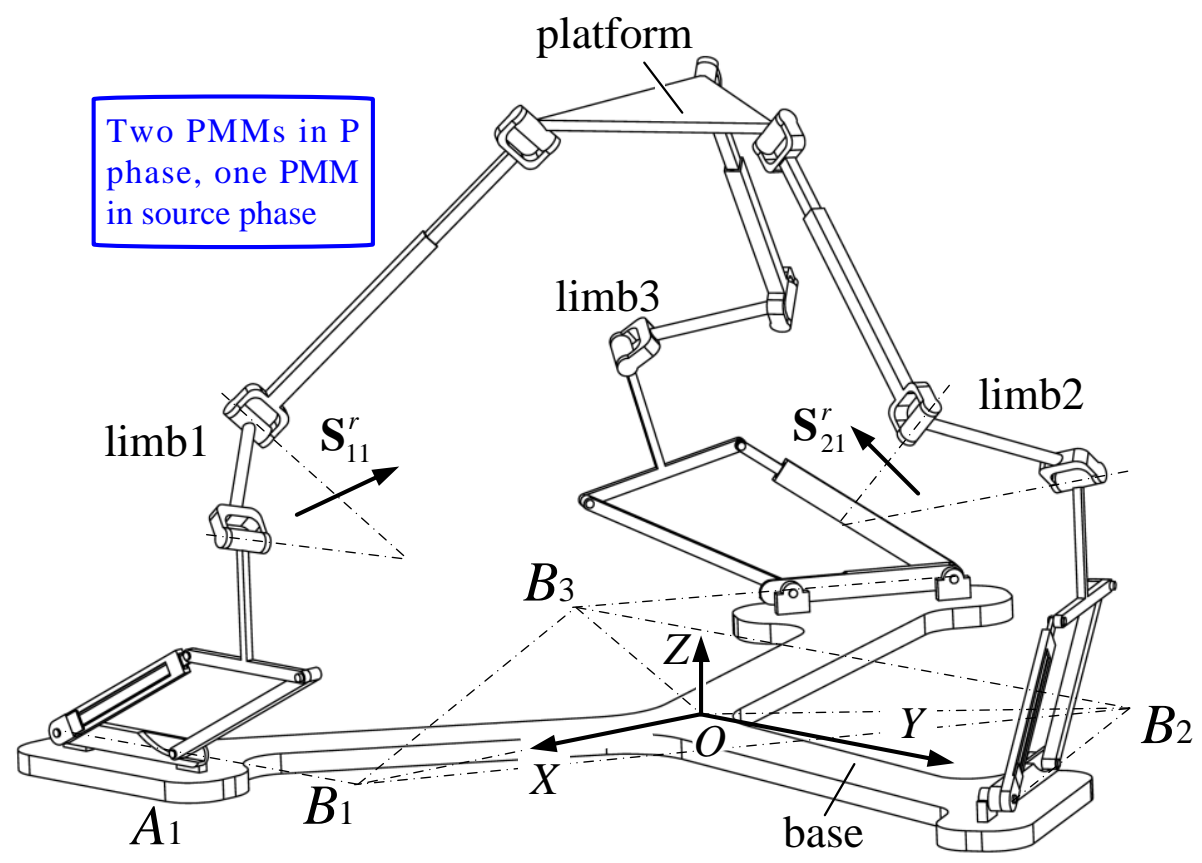

Fig.16

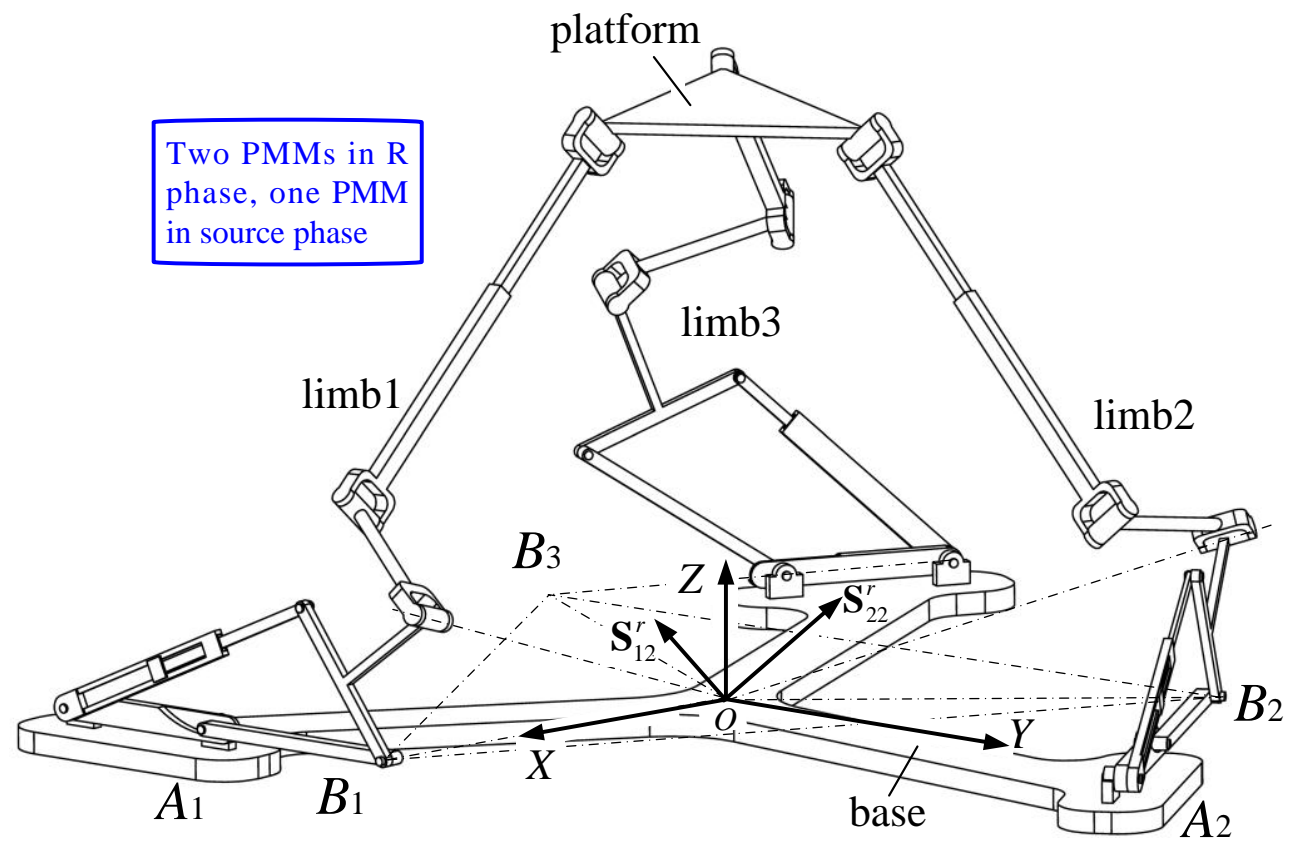

Fig.17 


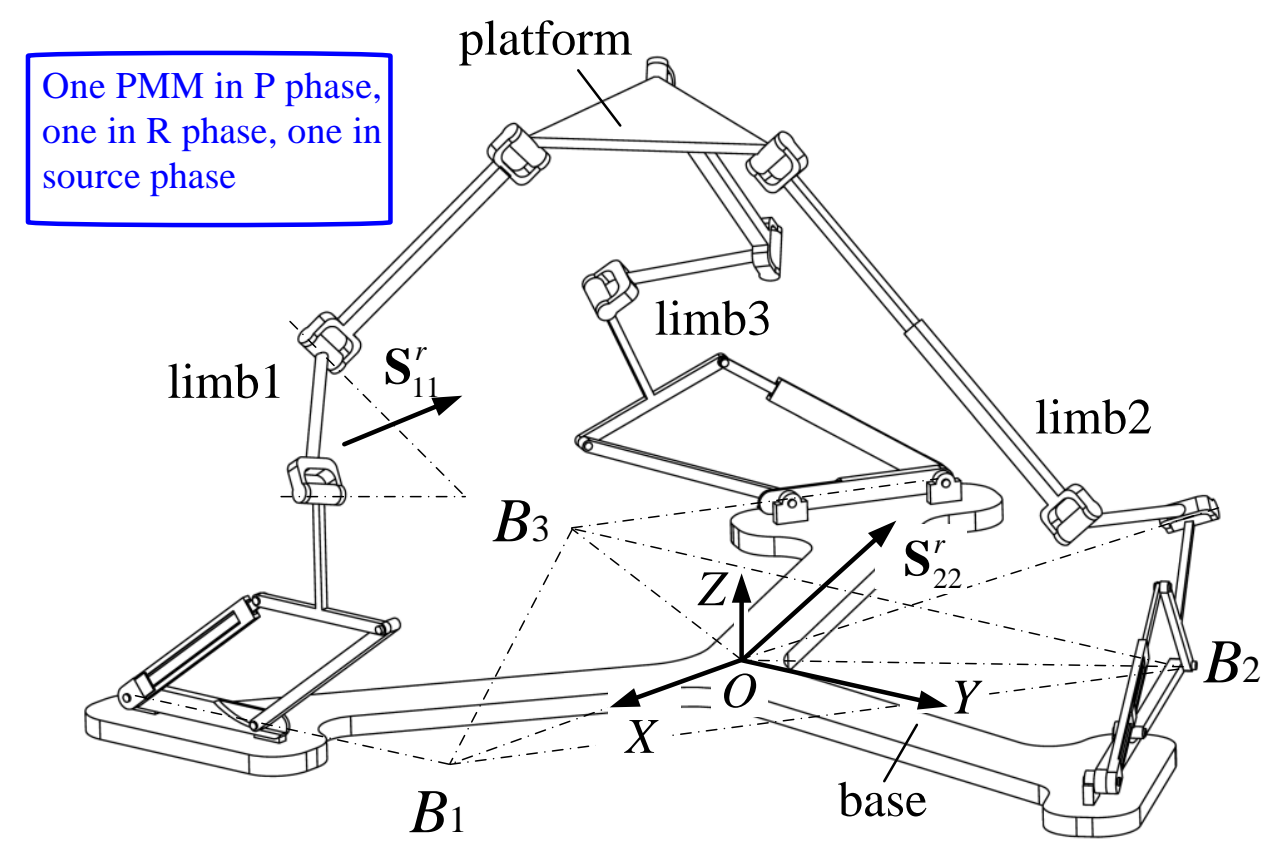

Fig. 18

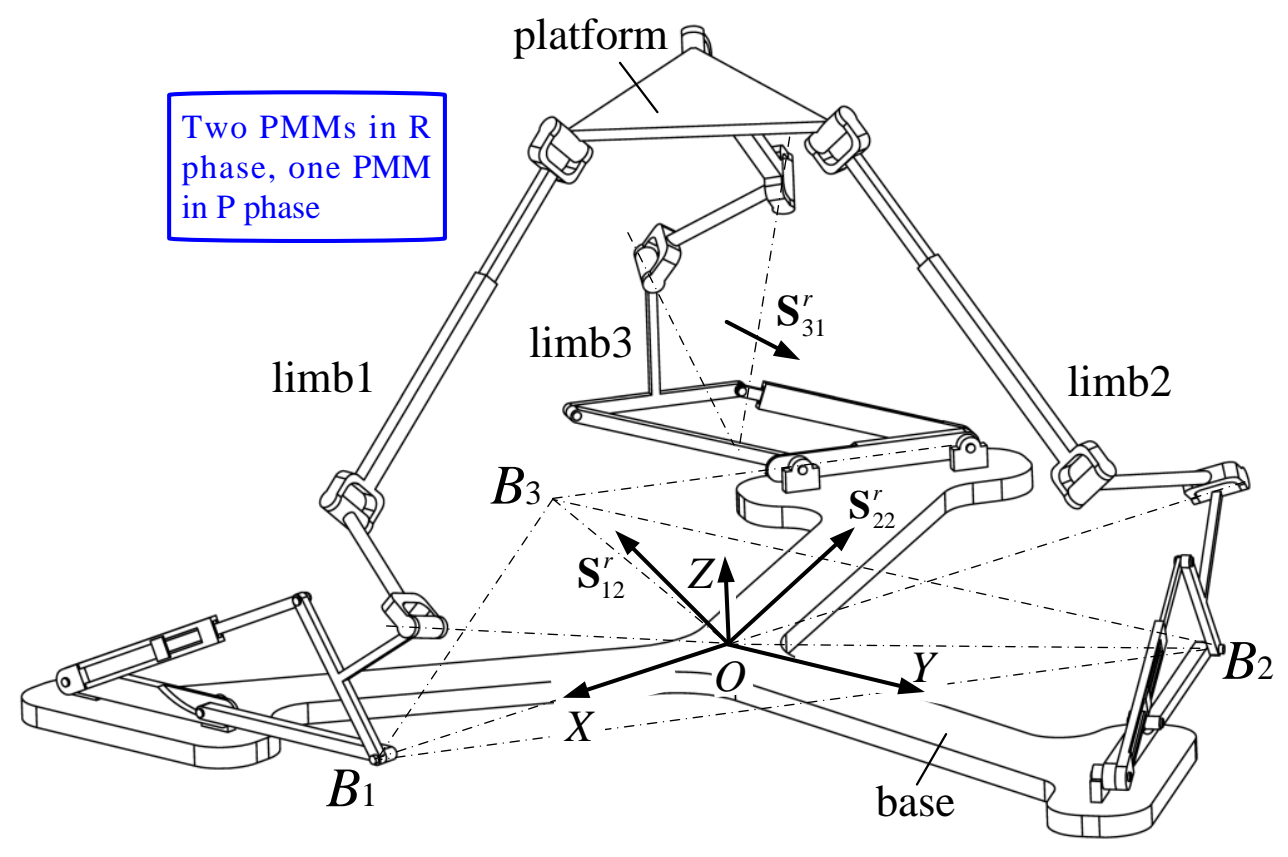

Fig.19 


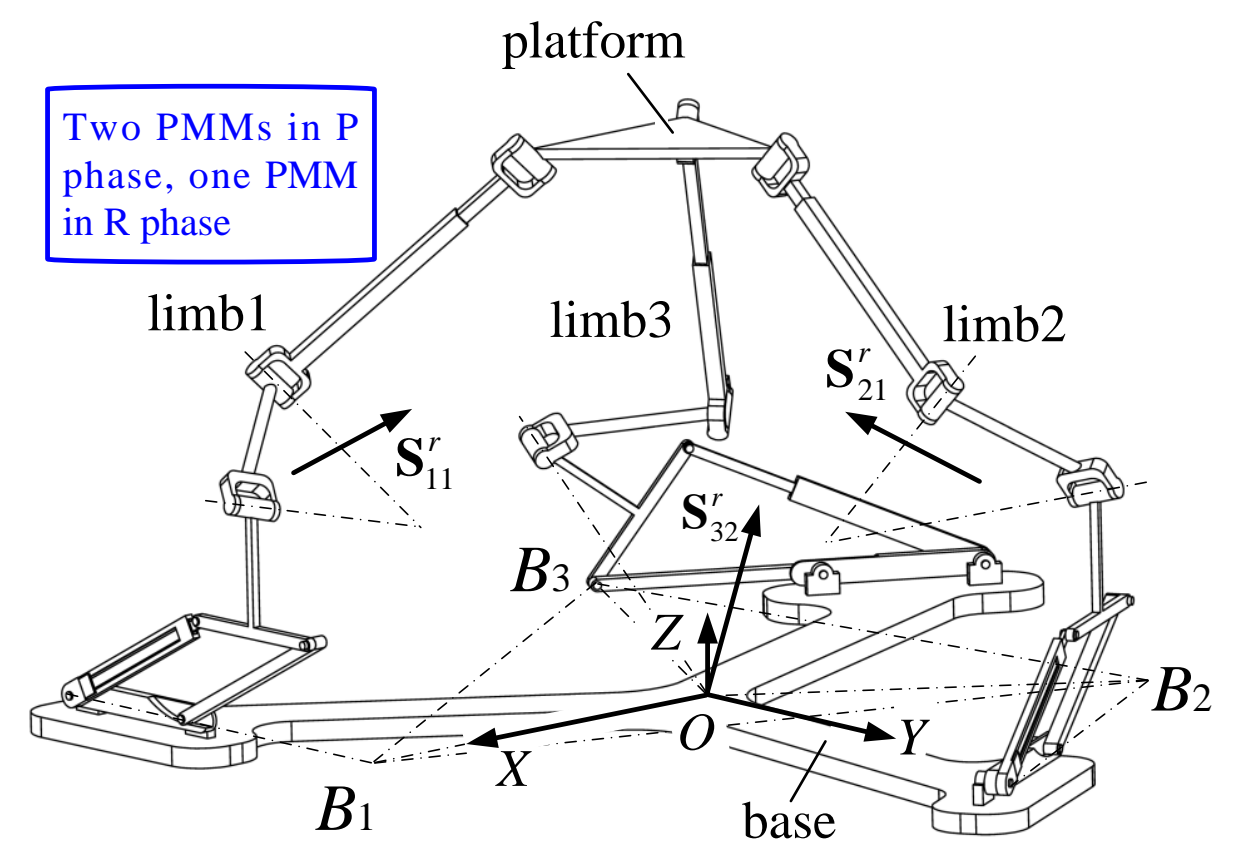

Fig.20

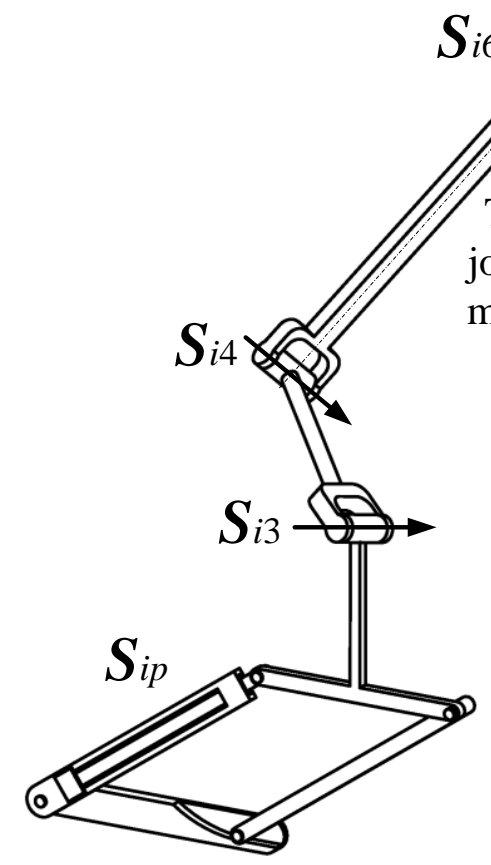

(a)
This prismatic

joint reaches the minimum stroke

base
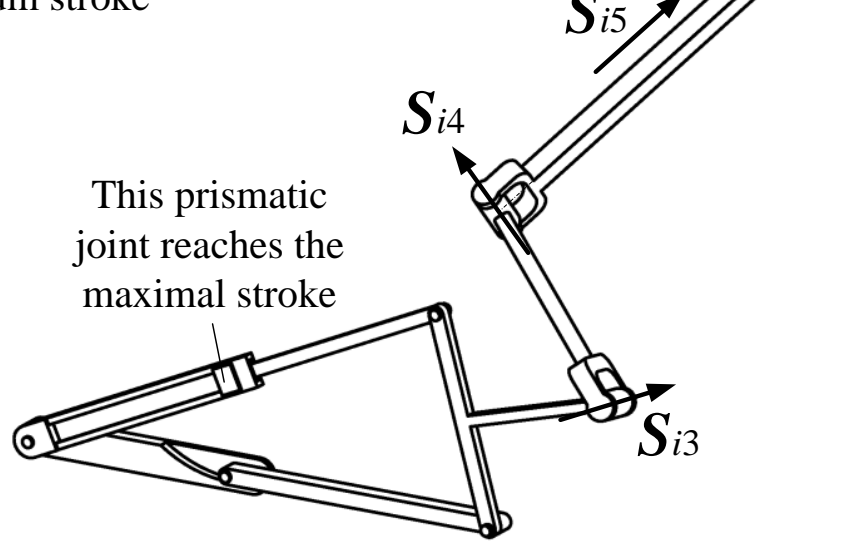

(b)

Fig.21 


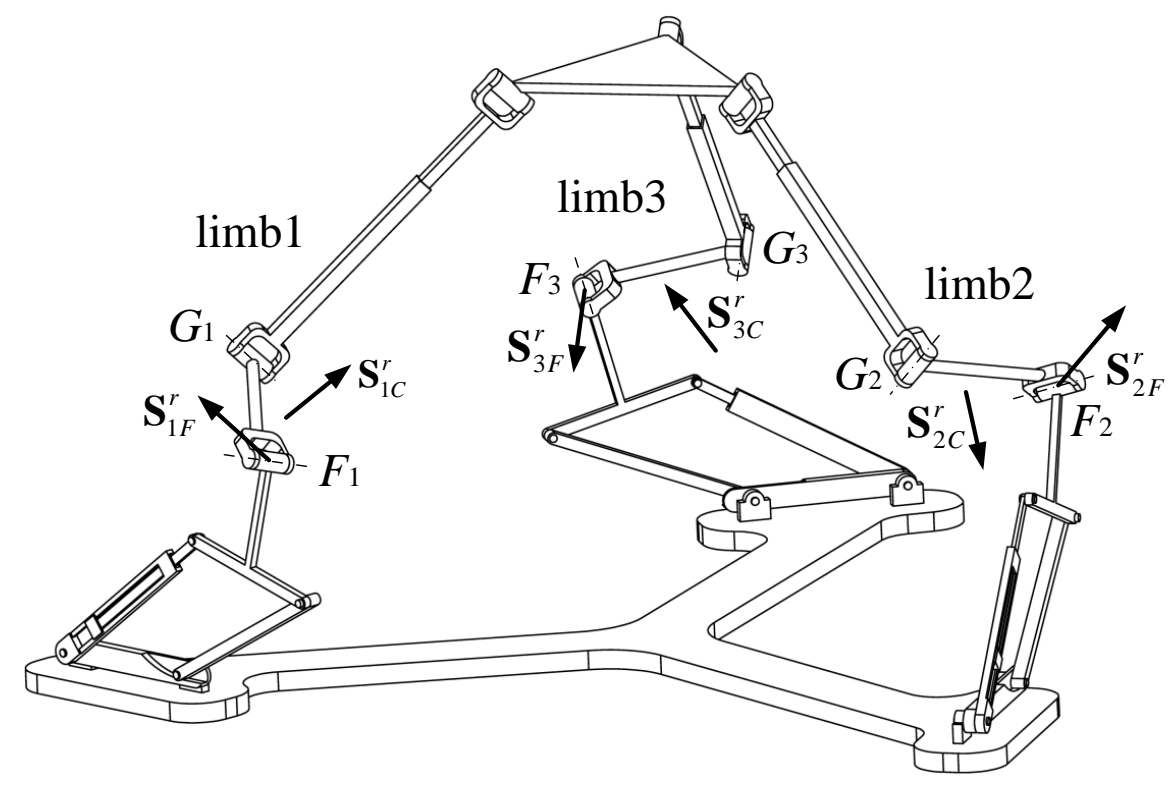

Fig.22

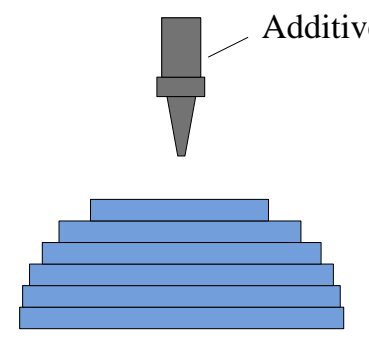

Additive manufacturing stage: three translational DOFs required

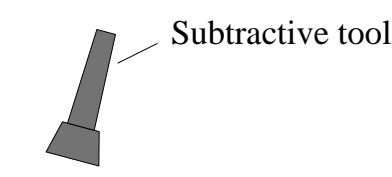

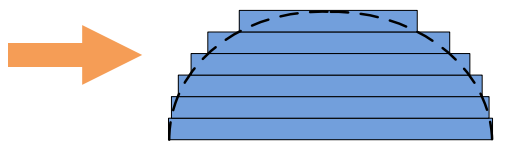

Subtractive manufacturing stage: three translational and two rotational DOFs required

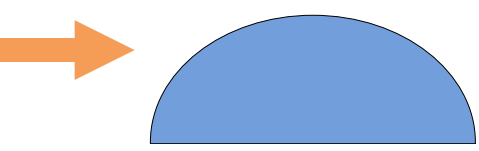

Final shape

Fig.23 